(n)

trobertivier Journal of Nonlinear Mathematical Physics

\title{
The Hankel determinant associated with a singularly perturbed
}

\section{Laguerre unitary ensemble}

Shulin Lyu, James Griffin, Yang Chen

To cite this article: Shulin Lyu, James Griffin, Yang Chen (2019) The Hankel determinant associated with a singularly perturbed Laguerre unitary ensemble, Journal of Nonlinear Mathematical Physics 26:1, 24-53, DOI:

https://doi.org/10.1080/14029251.2019.1544786

To link to this article: https://doi.org/10.1080/14029251.2019.1544786

Published online: 04 January 2021 


\title{
The Hankel determinant associated with a singularly perturbed Laguerre unitary ensemble
}

\author{
Shulin Lyu \\ School of Mathematics (Zhuhai), Sun Yat-sen University, \\ Zhuhai 519082, Guangdong, China \\ lvshulin1989@163.com \\ James Griffin \\ Department of Mathematics and Statistics, American University of Sharjah \\ Sharjah, PO Box 26666, UAE \\ jgriffin@aus.edu \\ Yang Chen \\ Department of Mathematics, University of Macau \\ Avenida da Universidade, Taipa, Macau, China \\ yangbrookchen@yahoo.co.uk
}

Received 24 January 2018

Accepted 24 May 2018

\begin{abstract}
We are concerned with the probability that all the eigenvalues of a unitary ensemble with the weight function $w(x ; t)=x^{\alpha} e^{-x-\frac{t}{x}}, x \in[0, \infty), \alpha>-1, t \geq 0$, are greater than $s$. This probability is expressed as the quotient of $D_{n}(s, t)$ and its value at $s=0$, where $D_{n}(s, t)$ denotes the determinant of the $n$ dimensional Hankel matrices generated by the moments of $w(x ; t)$ on $x \in[s, \infty)$. In this paper we focus specifically on the Hankel determinant $D_{n}(s, t)$ and its properties.

Based on the ladder operators adapted to the monic polynomials orthogonal with respect to $w(x ; t)$, and from the associated supplementary conditions and a sum-rule, we show that the log-derivative of the Hankel determinant, viewed as a function of $s$ and $t$, satisfies a second order sixth degree partial differential equation, where $n$ appears as a parameter. In order to go to the thermodynamic limit, of infinitely large matrices, we envisage a scenario where $n \rightarrow \infty, s \rightarrow 0$, and $t \rightarrow 0$ such that $S:=4 n s$ and $T:=(2 n+1+\alpha) t$ are finite. After such a double scaling, the large finite $n$ equation reduces to a second order second degree equation, in the variables $S$ and $T$, from which we derive the asymptotic expansion of the scaled Hankel determinant in three cases of $S$ and $T: S \rightarrow \infty$ with $T$ fixed, $S \rightarrow 0$ with $T>0$ fixed, and $T \rightarrow \infty$ with $S>0$ fixed. The constant term in the asymptotic expansion is shown to satisfy a difference equation and one of its solutions is the Tracy-Widom constant.
\end{abstract}

Keywords: Hankel Determinant; Smallest eigenvalue; Double scaling.

2000 Mathematics Subject Classification: 15B52, 42C05

\section{Introduction}

A unitary ensemble of Hermitian matrices $M=\left(M_{i j}\right)_{n \times n}$ has probability density

$$
\begin{aligned}
& p(M) d M \propto \mathrm{e}^{-\operatorname{tr} v(M)} \operatorname{vol}(\mathrm{dM}), \\
& \operatorname{vol}(\mathrm{dM})=\prod_{i=1}^{n} d M_{i i} \prod_{1 \leq j<k \leq n} d\left(\operatorname{Re} M_{j k}\right) d\left(\operatorname{Im} M_{j k}\right) .
\end{aligned}
$$


Here $v(M)$ is a matrix function [21] defined via Jordan canonical form and $v o l(\mathrm{dM})$ is called the volume element [22]. In this paper, we consider

$$
v(x)=v(x ; t)=-\alpha \log x+x+\frac{t}{x}, \quad x \in[0, \infty), \alpha>-1, t \geq 0 .
$$

Under an eigenvalue-eigenvector decomposition, the joint probability density function of the eigenvalues $\left\{x_{k}\right\}_{k=1}^{n}$ of this ensemble is given by [29]

$$
\frac{1}{D_{n}[w]} \frac{1}{n !} \prod_{1 \leq i<j \leq n}\left(x_{j}-x_{i}\right)^{2} \prod_{k=1}^{n} w\left(x_{k} ; t\right)
$$

where $D_{n}[w]$ is the normalization constant and $w(x ; t)$ is a positive weight function supported on $[0, \infty)$ and defined by

$$
w(x ; t):=\mathrm{e}^{-v(x ; t)}=x^{\alpha} \mathrm{e}^{-x-\frac{t}{x}}
$$

which has finite moments

$$
\mu_{k}:=\int_{0}^{\infty} x^{k} w(x ; t) d x, \quad k=0,1,2, \ldots
$$

Denote by $\mathbb{P}(s, t, n)$ the probability that all the eigenvalues of this ensemble are greater than $s$, then

$$
\begin{aligned}
\mathbb{P}(s, t, n)= & \frac{\frac{1}{n !} \int_{(s, \infty)^{n}} \prod_{1 \leq i<j \leq n}\left(x_{j}-x_{i}\right)^{2} \prod_{k=1}^{n} w\left(x_{k} ; t\right) d x_{k}}{\frac{1}{n !} \int_{(0, \infty)^{n}} \prod_{1 \leq i<j \leq n}\left(x_{j}-x_{i}\right)^{2} \prod_{k=1}^{n} w\left(x_{k} ; t\right) d x_{k}} \\
= & \frac{\operatorname{det}\left(\int_{s}^{\infty} x^{i+j} w(x ; t) d x\right)_{i, j=0}^{n-1}}{\operatorname{det}\left(\int_{0}^{\infty} x^{i+j} w(x ; t) d x\right)_{i, j=0}^{n-1}} \\
= & \frac{D_{n}(s, t)}{D_{n}(0, t)}
\end{aligned}
$$

Here note that the multiple integral is represented as the determinant of the Hankel (or moment) matrix. See [32].

The Hankel determinant is a fundamental object in random matrix theory [29]. It can be used to describe the eigenvalue distribution of the Gaussian, Laguerre and Jacobi unitary ensembles $[4,27,30]$ and also be applied to study the outage capacity and the error probability of multipleinput multiple-output antenna wireless communication systems $[9,15]$.

An elementary approach to dealing with the Hankel determinant is to write it as the product of the square of the $L^{2}$ norms of the non-standard orthogonal polynomials. From the supplementary conditions associated with the ladder operators for the orthogonal polynomials, we derive a series of difference and differential equations to characterize the Hankel determinant, usually involving a Painlevé equation or the associated $\sigma$ form. Such a formalism has been applied to the Hankel determinant generated by the deformation of the classical Gaussian weight $[8,16]$, the classical Laguerre weight $[1,2]$, and the (shifted) classical Jacobi weight $[3,7,17,18]$. 
When the order of the Hankel matrix, $n$, becomes large, one chooses a suitable combination of $n$ and a parameter present in the weight function such that the combination remains finite in the limit. Under such a double scaling, from the finite $n$ equations, one can derive a differential equation satisfied by the log-derivative of the scaled Hankel determinant in the scaled variable. From this equation and by integration, the asymptotic expansion of the scaled Hankel determinant is established. The constant term in the expansion is determined by using the linear statistics results [14] based on logarithmic potential theory with an external field, sometimes referred to in this context as Dyson's Coulomb fluid approximation [19]. See for example [5,6,27] for the formulation.

In this paper we shall apply the approach described above for finite $n$ and large $n$ to study the Hankel determinant

$$
D_{n}(s, t)=\operatorname{det}\left(\int_{s}^{\infty} x^{i+j} w(x ; t) d x\right)_{i, j=0}^{n-1},
$$

with $s \geq 0$, and with the weight function

$$
w(x ; t)=x^{\alpha} \mathrm{e}^{-x-\frac{t}{x}}, \quad \alpha>-1, \quad t \geq 0 .
$$

As mentioned above, $D_{n}(s, t)$ is connected with the smallest eigenvalue distribution of the singularly deformed Laguerre unitary ensemble. The singular deformation here means that the Laguerre weight or the Gamma density supported on $[0, \infty)$ is multiplied by a factor which vanishes infinitely fast at $x=0$, that is, $\mathrm{e}^{-\frac{t}{x}}, t>0$. The case $s=0$ and the case $t=0$ in $D_{n}(s, t)$ have been investigated for finite $n$ in [13] and [1] respectively through the ladder operator framework, and are discussed for large $n$ in [5] and [27] respectively by means of the double scaling and logarithmic potential theory with an external field. This two variable problem was suggested to the third author who spoke in a Seminar at the National Taiwan University on the $s=0$ problem. A person in the audience, suggested that the same weight supported on the interval $[s, \infty)$ maybe an interesting problem to study, since this would naturally lead to a PDE in the variables $s$ and $t$ which opens up a venue for the investigation of varying scaling limits between the 'gap' and the 'time' variables.

Let $h_{j}(s, t)$ be the square of the $L^{2}$ norm of monic polynomial $P_{j}(x ; s, t)$ orthogonal with respect to $w(x ; t)$ over $[s, \infty)$ :

$$
h_{j}(s, t) \delta_{j k}:=\int_{s}^{\infty} P_{j}(x ; s, t) P_{k}(x ; s, t) w(x ; t) d x, \quad j, k=0,1,2, \ldots,
$$

and define $p(j, s, t)$ by

$$
P_{j}(x ; s, t)=x^{j}+p(j, s, t) x^{j-1}+\cdots+P_{j}(0 ; s, t) .
$$

Then $D_{n}(s, t)$ admits a representation [32]

$$
D_{n}(s, t)=\prod_{j=0}^{n-1} h_{j}(s, t) .
$$

From the orthogonality relation, there follows the three-term recurrence relation

$$
z P_{n}(z ; s, t)=P_{n+1}(z ; s, t)+\alpha_{n}(s, t) P_{n}(z ; s, t)+\beta_{n}(s, t) P_{n-1}(z ; s, t), \quad n \geq 0,
$$

subject to the initial conditions

$$
P_{0}(z ; s, t):=1, \quad \beta_{0}(s, t) P_{-1}(z ; s, t):=0,
$$


and the recurrence coefficients are given by

$$
\alpha_{n}(s, t)=p(n, s, t)-p(n+1, s, t), \quad \beta_{n}(s, t)=\frac{h_{n}(s, t)}{h_{n-1}(s, t)} .
$$

As an immediate consequence, we have

$$
\sum_{j=0}^{n-1} \alpha_{j}(s, t)=-p(n, s, t)
$$

This paper is built up as follows. By using $\left(S_{1}\right)$ and $\left(S_{2}^{\prime}\right)$ (given later), we express in the next section the recurrence coefficients $\alpha_{n}(s, t)$ and $\beta_{n}(s, t)$ in terms of four auxiliary variables $R_{n}^{\star}(s, t)$, $R_{n}(s, t), r_{n}^{\star}(s, t)$ and $r_{n}(s, t)$, that satisfy a system of difference equations. Combining these expressions with the equalities obtained from the differentiation of (1.2) over $s$ and $t$, we establish a pair of second order partial differential equations satisfied by $R_{n}^{\star}(s, t)$ and $R_{n}(s, t)$, and a large second order sixth degree partial differential equation satisfied by $H_{n}(s, t):=\left(s \frac{\partial}{\partial s}+t \frac{\partial}{\partial t}\right) \log D_{n}(s, t)$. Based on these results for finite $n$, we devote Section 3 to the large $n$ analysis of $D_{n}(s, t)$ by assuming $n \rightarrow \infty$, $s \rightarrow 0$ and $t \rightarrow 0$ such that $S:=4 n s$ and $T:=(2 n+1+\alpha) t$ are fixed. We derive the asymptotic expansions of the scaled Hankel determinant in three cases of the new variables: $S$ is large or small with $T$ fixed, and $T$ is large with $S>0$ fixed. The constant term in the expansion for each case is shown to satisfy a difference equation in the last section, by using logarithmic potential theory with an external field to evaluate $P_{n}(z ; s, t)$ at $z=0$.

\section{Difference Equations and Differential Equations}

With the potential $v(x)=v(x ; t)$ given by

$$
v(x)=-\alpha \log x+x+\frac{t}{x},
$$

the monic polynomials $\left\{P_{n}(x ; s, t)\right\}$ orthogonal with respect to $w(x ; t)$ over $[s, \infty)$ satisfy a pair of ladder operators which lower or raise the index $n$ of $P_{n}(x ; s, t)$ :

$$
\begin{aligned}
\left(\frac{\partial}{\partial z}+B_{n}(z)\right) P_{n}(z ; s, t) & =\beta_{n}(s, t) A_{n}(z) P_{n-1}(z ; s, t), \\
\left(\frac{\partial}{\partial z}-B_{n}(z)-v^{\prime}(z)\right) P_{n-1}(z ; s, t) & =-A_{n-1}(z) P_{n}(z ; s, t),
\end{aligned}
$$

where $A_{n}(z)=A_{n}(z ; s, t)$ and $B_{n}(z)=B_{n}(z ; s, t)$ are defined by

$$
\begin{aligned}
A_{n}(z):= & \left.\frac{P_{n}^{2}(y ; s, t) w(y ; t)}{h_{n}(s, t)(y-z)}\right|_{y=s} ^{y=\infty} \\
& +\frac{1}{h_{n}(s, t)} \int_{s}^{\infty} \frac{v^{\prime}(z)-v^{\prime}(y)}{z-y} P_{n}^{2}(y ; s, t) w(y ; t) d y, \\
B_{n}(z):= & \left.\frac{P_{n}(y ; s, t) P_{n-1}(y ; s, t) w(y ; t)}{h_{n-1}(s, t)(y-z)}\right|_{y=s} ^{y=\infty} \\
& +\frac{1}{h_{n-1}(s, t)} \int_{s}^{\infty} \frac{v^{\prime}(z)-v^{\prime}(y)}{z-y} P_{n}(y ; s, t) P_{n-1}(y ; s, t) w(y ; t) d y .
\end{aligned}
$$


From the above ladder operators and the recurrence relation for $P_{n}(z ; s, t)$, one can derive two compatibility conditions satisfied by $A_{n}(z)$ and $B_{n}(z)$ :

$$
\begin{aligned}
B_{n+1}(z)+B_{n}(z) & =\left(z-\alpha_{n}(s, t)\right) A_{n}(z)-v^{\prime}(z), \\
1+\left(z-\alpha_{n}(s, t)\right)\left(B_{n+1}(z)-B_{n}(z)\right) & =\beta_{n+1}(s, t) A_{n+1}(z)-\beta_{n}(s, t) A_{n-1}(z) .
\end{aligned}
$$

$\left(S_{1}\right)$ and $\left(S_{2}\right)$ can be combined to give the 'sum rule':

$$
B_{n}^{2}(z)+v^{\prime}(z) B_{n}(z)+\sum_{j=0}^{n-1} A_{j}(z)=\beta_{n}(s, t) A_{n}(z) A_{n-1}(z) .
$$

Instrumental for the deductions that follow. See $[11,12,28]$ for a detailed derivation. See also [23, $\S 3.2]$ for the form adapted to orthonormal polynomials.

For our problem, we have

$$
\frac{v^{\prime}(z)-v^{\prime}(y)}{z-y}=\frac{\alpha}{z y}+\frac{t}{z y^{2}}+\frac{t}{z^{2} y} .
$$

Hence, from the definitions of $A_{n}(z)$ and $B_{n}(z)$, and through integration by parts, we obtain

$$
\begin{aligned}
& A_{n}(z)=\frac{R_{n}^{\star}(s, t)}{z-s}+\frac{1-R_{n}^{\star}(s, t)}{z}+\frac{t R_{n}(s, t)}{z^{2}}, \\
& B_{n}(z)=\frac{r_{n}^{\star}(s, t)}{z-s}-\frac{r_{n}^{\star}(s, t)+n}{z}+\frac{t r_{n}(s, t)}{z^{2}} .
\end{aligned}
$$

Here the auxiliary quantities $R_{n}^{\star}(s, t), R_{n}(s, t), r_{n}^{\star}(s, t)$ and $r_{n}(s, t)$ are defined by

$$
\begin{aligned}
R_{n}^{\star}(s, t) & :=\frac{w(s ; t)}{h_{n}(s, t)} P_{n}^{2}(s ; s, t), \\
R_{n}(s, t) & :=\frac{1}{h_{n}(s, t)} \int_{s}^{\infty} \frac{d y}{y} P_{n}^{2}(y ; s, t) w(y ; t), \\
r_{n}^{\star}(s, t) & :=\frac{w(s ; t)}{h_{n-1}(s, t)} P_{n}(s ; s, t) P_{n-1}(s ; s, t), \\
r_{n}(s, t) & :=\frac{1}{h_{n-1}(s, t)} \int_{s}^{\infty} \frac{d y}{y} P_{n}(y ; s, t) P_{n-1}(y ; s, t) w(y ; t) .
\end{aligned}
$$

Note that $P_{n}(s ; s, t)$ is the evaluation of $P_{n}(z ; s, t)$ at $z=s$ and $w(s ; t)=s^{\alpha} \mathrm{e}^{-s-\frac{t}{s}}$.

\subsection{The recurrence coefficients in terms of the auxiliary quantities}

Substituting (2.1) into $\left(S_{1}\right)$ and $\left(S_{2}^{\prime}\right)$, and by equating the residues, we can establish a set of equations that will give insight into the recurrence coefficients $\alpha_{n}(s, t), \beta_{n}(s, t)$ and the auxiliary quantities.

From $\left(S_{1}\right)$, we arrive at three difference equations relating $\alpha_{n}(s, t)$ to the auxiliary quantities:

$$
\begin{aligned}
r_{n+1}^{\star}+r_{n}^{\star} & =\left(s-\alpha_{n}\right) R_{n}^{\star}, \\
r_{n+1}+r_{n} & =1-\alpha_{n} R_{n}, \\
r_{n+1}^{\star}+r_{n}^{\star}+2 n+1 & =\alpha_{n}\left(1-R_{n}^{\star}\right)-t R_{n}-\alpha .
\end{aligned}
$$


From $\left(S_{2}^{\prime}\right)$, we obtain the following difference equations involving $\beta_{n}, \sum_{j=0}^{n-1} R_{j}, \sum_{j=0}^{n-1} R_{j}^{\star}$, and the auxiliary quantities:

$$
\begin{gathered}
\left(r_{n}^{\star}\right)^{2}=\beta_{n} R_{n}^{\star} R_{n-1}^{\star}, \\
r_{n}^{2}-r_{n}=\beta_{n} R_{n} R_{n-1}, \\
n-(2 n+\alpha) r_{n}-2 r_{n} r_{n}^{\star}+r_{n}^{\star}=\beta_{n}\left(R_{n}\left(1-R_{n-1}^{\star}\right)+R_{n-1}\left(1-R_{n}^{\star}\right)\right), \\
\left(r_{n}^{\star}+n\right)^{2}-\frac{2 t r_{n} r_{n}^{\star}}{s}+t r_{n}+\frac{t r_{n}^{\star}}{s}+\alpha\left(r_{n}^{\star}+n\right)+t \sum_{j=0}^{n-1} R_{j} \\
=\beta_{n}\left(1-R_{n}^{\star}\right)\left(1-R_{n-1}^{\star}\right)-\frac{\beta_{n} t R_{n} R_{n-1}^{\star}-\frac{\beta_{n} t R_{n}^{\star} R_{n-1}}{s},}{s} \\
-2 r_{n}^{\star}\left(r_{n}^{\star}+n\right)+\frac{2 t r_{n} r_{n}^{\star}}{s}+s r_{n}^{\star}-\frac{t r_{n}^{\star}}{s}-\alpha r_{n}^{\star}+s \sum_{j=0}^{n-1} R_{j}^{\star} \\
=\beta_{n} R_{n}^{\star}\left(1-R_{n-1}^{\star}\right)+\beta_{n} R_{n-1}^{\star}\left(1-R_{n}^{\star}\right)+\frac{\beta_{n} t R_{n} R_{n-1}^{\star}}{s}+\frac{\beta_{n} t R_{n}^{\star} R_{n-1}}{s} .
\end{gathered}
$$

To continue, we make use of (2.2)-(2.7) to show that $\alpha_{n}(s, t)$ and $\beta_{n}(s, t)$ are expressible in terms of the auxiliary quantities and in turn satisfy a system of difference equations.

Lemma 2.1. The recurrence coefficients $\alpha_{n}(s, t)$ and $\beta_{n}(s, t)$ are expressed in terms of $R_{n}^{\star}(s, t), R_{n}(s, t), r_{n}^{\star}(s, t)$, and $r_{n}(s, t)$ by

$$
\begin{gathered}
\alpha_{n}(s, t)=2 n+1+\alpha+s R_{n}^{\star}+t R_{n}, \\
\beta_{n}(s, t)=\frac{1}{R_{n}}\left(n-(2 n+\alpha) r_{n}-2 r_{n} r_{n}^{\star}+r_{n}^{\star}\right)-\frac{r_{n}^{2}-r_{n}}{R_{n}^{2}}\left(1-R_{n}^{\star}\right)+\frac{\left(r_{n}^{\star}\right)^{2}}{R_{n}^{\star}} .
\end{gathered}
$$

Proof. Subtracting (2.2) from (2.4) leads to (2.10). Eliminating $R_{n-1}^{\star}$ and $R_{n-1}$ from (2.7) by using (2.5) and (2.6) results in (2.11).

Proposition 2.1. The auxiliary quantities $R_{n}^{\star}(s, t), R_{n}(s, t), r_{n}^{\star}(s, t)$ and $r_{n}(s, t)$ satisfy the following difference equations

$$
\begin{gathered}
r_{n+1}^{\star}+r_{n}^{\star}=\left(s-\left(2 n+1+\alpha+s R_{n}^{\star}+t R_{n}\right)\right) R_{n}^{\star}, \\
r_{n+1}+r_{n}=1-\left(2 n+1+\alpha+s R_{n}^{\star}+t R_{n}\right) R_{n}, \\
\left(r_{n}^{2}-r_{n}\right) R_{n}^{\star} R_{n-1}^{\star}=\left(r_{n}^{\star}\right)^{2} R_{n} R_{n-1}, \\
\left(r_{n}^{\star}\right)^{2}\left(R_{n}\left(1-R_{n-1}^{\star}\right)+R_{n-1}\left(1-R_{n}^{\star}\right)\right)=\left(n-(2 n+\alpha) r_{n}-2 r_{n} r_{n}^{\star}+r_{n}^{\star}\right) R_{n}^{\star} R_{n-1}^{\star},
\end{gathered}
$$

which can be solved with the initial conditions

$$
R_{0}^{\star}(s, t)=\frac{s^{\alpha} \mathrm{e}^{-s-\frac{t}{s}}}{\int_{s}^{\infty} y^{\alpha} \mathrm{e}^{-y-\frac{t}{y}} d y}, \quad R_{0}(s, t)=\frac{\int_{s}^{\infty} y^{\alpha-1} \mathrm{e}^{-y-\frac{t}{y}} d y}{\int_{s}^{\infty} y^{\alpha} \mathrm{e}^{-y-\frac{t}{y}} d y}, \quad r_{0}^{\star}(s, t)=0, \quad r_{0}(s, t)=0 .
$$


Proof. Substituting (2.10) into (2.2) and (2.3) gives rise to (2.12) and (2.13) respectively. Getting rid of $\beta_{n}$ from (2.5) and (2.6), we get (2.14). Eliminating $\beta_{n}$ from (2.7) by using (2.5) yields (2.15).

Finally, we present two expressions involving $\sum_{j=0}^{n-1}\left(s R_{j}^{\star}(s, t)+t R_{j}(s, t)\right)$, which we will see are crucial for the forthcoming derivations.

Lemma 2.2. We have

$$
\begin{aligned}
-\sum_{j=0}^{n-1}\left(s R_{j}^{\star}(s, t)+t R_{j}(s, t)\right) & =p(n, s, t)+n(n+\alpha) \\
& =s r_{n}^{\star}(s, t)+t r_{n}(s, t)-\beta_{n}(s, t)+n(n+\alpha),
\end{aligned}
$$

and a combination of (2.16) and (2.17) gives rise to

$$
\beta_{n}(s, t)=s r_{n}^{\star}(s, t)+t r_{n}(s, t)-p(n, s, t) .
$$

Proof. Replacing $n$ by $j$ in (2.10), we have

$$
\alpha_{j}(s, t)=2 j+1+\alpha+s R_{j}^{\star}(s, t)+t R_{j}(s, t) .
$$

Summing it from $j=0$ to $n-1$, in view of $\sum_{j=0}^{n-1} \alpha_{j}(s, t)=-p(n, s, t)$, we come to (2.16). Equation (2.17) results from the summation of (2.8) and (2.9).

\section{2. s evolution and t evolution}

We shall consider the differentiation of

$$
h_{j}(s, t) \delta_{j k}:=\int_{s}^{\infty} P_{j}(x ; s, t) P_{k}(x ; s, t) w(x ; t) d x,
$$

with $s$ and $t$, with $j=k=n$ and $j=k+1=n$. We see that $R_{n}^{\star}(s, t)$ and $R_{n}(s, t)$ are closely related to the log-derivative of $h_{n}(s, t)$, while $r_{n}^{\star}(s, t)$ and $r_{n}(s, t)$ are connected with the derivative of $p(n, s, t)$. With the aid of the identities in the previous subsection, we derive a system of first order partial differential equations satisfied by $R_{n}^{\star}(s, t), R_{n}(s, t), r_{n}^{\star}(s, t)$ and $r_{n}(s, t)$.

Taking the derivative of

$$
h_{n}(s, t)=\int_{s}^{\infty} P_{n}^{2}(x ; s, t) w(x ; t) d x
$$

with respect to $s$ and $t$, we get

$$
\begin{aligned}
& \partial_{s} \log h_{n}(s, t)=-R_{n}^{\star}(s, t), \\
& \partial_{t} \log h_{n}(s, t)=-R_{n}(s, t) .
\end{aligned}
$$

From the fact $\beta_{n}(s, t)=h_{n}(s, t) / h_{n-1}(s, t)$ and (2.19), it follows that

$$
\begin{aligned}
& \partial_{s} \log \beta_{n}(s, t)=R_{n-1}^{\star}(s, t)-R_{n}^{\star}(s, t), \\
& \partial_{t} \log \beta_{n}(s, t)=R_{n-1}(s, t)-R_{n}(s, t) .
\end{aligned}
$$


Shulin Lyu et al. / The Hankel determinant associated with a singularly perturbed Laguerre unitary ensemble

We proceed with the differentiation with $s$ and $t$ on

$$
0=\int_{s}^{\infty} P_{n}(x ; s, t) P_{n-1}(x ; s, t) w(x ; t) d x,
$$

to find,

$$
\begin{aligned}
& \partial_{s} p(n, s, t)=r_{n}^{\star}(s, t), \\
& \partial_{t} p(n, s, t)=r_{n}(s, t) .
\end{aligned}
$$

Hence, in view of $\alpha_{n}(s, t)=p(n, s, t)-p(n+1, s, t)$, we find

$$
\begin{aligned}
& \partial_{s} \alpha_{n}(s, t)=r_{n}^{\star}(s, t)-r_{n+1}^{\star}(s, t), \\
& \partial_{t} \alpha_{n}(s, t)=r_{n}(s, t)-r_{n+1}(s, t) .
\end{aligned}
$$

Moreover, on combining (2.18) with (2.21), we come to a representation of $\beta_{n}(s, t)$ in terms of $p(n, s, t)$ and its first order partial derivatives:

$$
\beta_{n}(s, t)=\left(s \partial_{s}+t \partial_{t}\right) p(n, s, t)-p(n, s, t) .
$$

Now, we are in a position to derive analogs of coupled Riccati equations: There are four equations instead of two.

Lemma 2.3. The auxiliary quantities $R_{n}^{\star}(s, t), R_{n}(s, t), r_{n}^{\star}(s, t)$ and $r_{n}(s, t)$ satisfy four first order partial differential equations

$$
\begin{aligned}
s \partial_{s} R_{n}^{\star}+t \partial_{s} R_{n}=2 r_{n}^{\star}+\left(2 n+\alpha+s R_{n}^{\star}+t R_{n}\right) R_{n}^{\star}-s R_{n}^{\star}, \\
s \partial_{t} R_{n}^{\star}+t \partial_{t} R_{n}=2 r_{n}+\left(2 n+\alpha+s R_{n}^{\star}+t R_{n}\right) R_{n}-1, \\
s\left(\partial_{s} r_{n}^{\star}\right)+t\left(\partial_{s} r_{n}\right)=\left(1-R_{n}^{\star}\right)\left(\frac{\left(r_{n}^{\star}\right)^{2}}{R_{n}^{\star}}+\frac{R_{n}^{\star}}{R_{n}^{2}}\left(r_{n}^{2}-r_{n}\right)\right) \\
+\frac{R_{n}^{\star}}{R_{n}}\left(2 r_{n} r_{n}^{\star}-r_{n}^{\star}+(2 n+\alpha) r_{n}-n\right), \\
s \partial_{t} r_{n}^{\star}+\partial_{t} r_{n}=-\frac{R_{n}}{R_{n}^{\star}}\left(r_{n}^{\star}\right)^{2}+\frac{2-R_{n}^{\star}}{R_{n}}\left(r_{n}^{2}-r_{n}\right) \\
+2 r_{n} r_{n}^{\star}-r_{n}^{\star}+(2 n+\alpha) r_{n}-n .
\end{aligned}
$$

Proof. We differentiate (2.10), i.e.

$$
\alpha_{n}(s, t)=2 n+1+\alpha+s R_{n}^{\star}(s, t)+t R_{n}(s, t),
$$

over $s$ and $t$ and in view of (2.22), to find,

$$
\begin{aligned}
& r_{n}^{\star}-r_{n+1}^{\star}=R_{n}^{\star}+s \partial_{s} R_{n}^{\star}+t \partial_{s} R_{n}, \\
& r_{n}-r_{n+1}=R_{n}+s \partial_{t} R_{n}^{\star}+t \partial_{t} R_{n} .
\end{aligned}
$$

Getting rid of $r_{n+1}^{\star}$ and $r_{n+1}$ by using (2.12) and (2.13) respectively, we are led to (2.24) and (2.25). 
Shulin Lyu et al. / The Hankel determinant associated with a singularly perturbed Laguerre unitary ensemble

Taking the derivative of (2.18), i.e.

$$
\beta_{n}(s, t)=s r_{n}^{\star}(s, t)+t r_{n}(s, t)-p(n, s, t),
$$

with respect to $s$ and $t$, and remembering that $\partial_{s} p(n, s, t)=r_{n}^{\star}(s, t)$ and $\partial_{t} p(n, s, t)=r_{n}(s, t)$, we find

$$
\begin{aligned}
& \beta_{n} R_{n-1}^{\star}-\beta_{n} R_{n}^{\star}=s \partial_{s} r_{n}^{\star}+t \partial_{s} r_{n}, \\
& \beta_{n} R_{n-1}-\beta_{n} R_{n}=s \partial_{t} r_{n}^{\star}+t \partial_{t} r_{n} .
\end{aligned}
$$

According to (2.5) and (2.6), we replace $\beta_{n} R_{n-1}^{\star}$ by $\frac{\left(r_{n}^{\star}\right)^{2}}{R_{n}^{\star}}$ and $\beta_{n} R_{n-1}$ by $\frac{r_{n}^{2}-r_{n}}{R_{n}}$ in the above two equations, then on removing $\beta_{n}$ by using (2.11) in the resulting equations, we arrive at (2.26) and (2.27).

We now look at the Hankel determinant in question, namely,

$$
\begin{aligned}
D_{n}(s, t) & =\operatorname{det}\left(\int_{s}^{\infty} x^{i+j} w(x ; t) d x\right)_{i, j=0}^{n-1} \\
& =\prod_{j=0}^{n-1} h_{j}(s, t) .
\end{aligned}
$$

Applying $s \partial_{s}+t \partial_{t}$ to

$$
\log D_{n}(s, t)=\sum_{j=0}^{n-1} \log h_{j}(s, t)
$$

we get, because of (2.19),

$$
\begin{aligned}
\left(s \partial_{s}+t \partial_{t}\right) \log D_{n}(s, t) & =-\sum_{j=0}^{n-1}\left(s R_{j}^{\star}(s, t)+t R_{j}(s, t)\right) \\
& =p(n, s, t)+n(n+\alpha),
\end{aligned}
$$

where the second equality comes from (2.16). Applying $s \partial_{s}+t \partial_{t}$ again to (2.29), in view of (2.21), we find

$$
\left(s^{2} \partial_{s s}+2 s t \partial_{s t}+t^{2} \partial_{t t}\right) \log D_{n}(s, t)+\left(s \partial_{s}+t \partial_{t}\right) \log D_{n}(s, t)=s r_{n}^{\star}(s, t)+t r_{n}(s, t),
$$

which combined with (2.29) yields

$$
\begin{aligned}
\left(s^{2} \partial_{s s}+2 s t \partial_{s t}+t^{2} \partial_{t t}\right) \log D_{n}(s, t) & =s r_{n}^{\star}(s, t)+t r_{n}(s, t)-p(n, s, t)-n(n+\alpha) \\
& =\beta_{n}(s, t)-n(n+\alpha),
\end{aligned}
$$

where the second equality is due to (2.18). Noting that

$$
\beta_{n}(s, t)=\frac{D_{n+1}(s, t) D_{n-1}(s, t)}{\left(D_{n}(s, t)\right)^{2}},
$$

which is a consequence of $\beta_{n}(s, t)=h_{n}(s, t) / h_{n-1}(s, t)$ and $h_{n}(s, t)=D_{n+1}(s, t) / D_{n}(s, t)$, we finally establish the following statement from (2.30) which can be viewed as a two variable version of the Toda-molecule equations. 
Shulin Lyu et al. / The Hankel determinant associated with a singularly perturbed Laguerre unitary ensemble

Proposition 2.2. The following second order partial differential-difference equation holds:

$$
\left(s^{2} \partial_{s s}+2 s t \partial_{s t}+t^{2} \partial_{t t}\right) \log D_{n}(s, t)=\frac{D_{n+1}(s, t) D_{n-1}(s, t)}{\left(D_{n}(s, t)\right)^{2}}-n(n+\alpha) .
$$

By making the substitution

$$
D_{n}(s, t)=(s+t)^{n(n+\alpha)} \widehat{D}_{n}(s, t),
$$

this is transformed into

$$
\left(s^{2} \partial_{s s}+2 s t \partial_{s t}+t^{2} \partial_{t t}\right) \log \widehat{D}_{n}(s, t)=(s+t)^{2} \frac{\widehat{D}_{n+1}(s, t) \widehat{D}_{n-1}(s, t)}{\left(\widehat{D}_{n}(s, t)\right)^{2}}
$$

To close this subsection, we apply (2.10), (2.20) and (2.22), namely the expressions involving the recurrence coefficients and their first order partial derivatives, to derive the results below.

Proposition 2.3. The following partial differential relations hold for the recurrence coefficients $\alpha_{n}(s, t)$ and $\beta_{n}(s, t)$ :

$$
\begin{aligned}
\left(s \partial_{s}+t \partial_{t}-1\right) \alpha_{n}(s, t) & =\beta_{n}(s, t)-\beta_{n+1}(s, t), \\
\left(s \partial_{s}+t \partial_{t}\right) \log \beta_{n}(s, t) & =\alpha_{n-1}(s, t)-\alpha_{n}(s, t)+2,
\end{aligned}
$$

and, as a consequence, we have

$$
\left(s^{2} \partial_{s s}+2 s t \partial_{s t}+t^{2} \partial_{t t}\right) \log \beta_{n}(s, t)=\beta_{n-1}(s, t)-2 \beta_{n}(s, t)+\beta_{n+1}(s, t)-2 .
$$

Proof. To prove (2.32), we apply $s \partial_{s}+t \partial_{t}$ to

$$
\alpha_{n}(s, t)=p(n, s, t)-p(n+1, s, t) .
$$

According to (2.21), we get

$$
\begin{aligned}
\left(s \partial_{s}+t \partial_{t}\right) \alpha_{n}(s, t) & =s r_{n}^{\star}(s, t)+t r_{n}(s, t)-\left(s r_{n+1}^{\star}(s, t)+t r_{n+1}(s, t)\right) \\
& =\beta_{n}(s, t)+p(n, s, t)-\left(\beta_{n+1}(s, t)+p(n+1, s, t)\right) \\
& =\beta_{n}(s, t)-\beta_{n+1}(s, t)+\alpha_{n}(s, t),
\end{aligned}
$$

where the second equality results from (2.18).

From (2.20), it follows that

$$
\left(s \partial_{s}+t \partial_{t}\right) \log \beta_{n}(s, t)=s R_{n-1}^{\star}(s, t)+t R_{n-1}(s, t)-\left(s R_{n}^{\star}(s, t)+t R_{n}(s, t)\right),
$$

which, in view of (2.10), leads to (2.33).

We differentiate (2.33) over $s$ and $t$ to obtain

$$
\begin{aligned}
& \left(\partial_{s}+s \partial_{s s}+t \partial_{s t}\right) \log \beta_{n}(s, t)=\partial_{s} \alpha_{n-1}(s, t)-\partial_{s} \alpha_{n}(s, t), \\
& \left(s \partial_{t s}+\partial_{t}+t \partial_{t t}\right) \log \beta_{n}(s, t)=\partial_{t} \alpha_{n-1}(s, t)-\partial_{t} \alpha_{n}(s, t) .
\end{aligned}
$$

Multiplying the first equality by $s$ and the second by $t$, and adding them, we find

$$
\begin{array}{r}
\left(s^{2} \partial_{s s}+2 s t \partial_{s t}+t^{2} \partial_{t t}\right) \log \beta_{n}(s, t)+\left(s \partial_{s}+t \partial_{t}\right) \log \beta_{n}(s, t) \\
=\left(s \partial_{s}+t \partial_{t}\right) \alpha_{n-1}(s, t)-\left(s \partial_{s}+t \partial_{t}\right) \alpha_{n}(s, t),
\end{array}
$$

which, combined with equations (2.32) and (2.33), establishes (2.34). 
Shulin Lyu et al. / The Hankel determinant associated with a singularly perturbed Laguerre unitary ensemble

\subsection{Partial differential equations satisfied by $R_{n}^{\star}(s, t), R_{n}(s, t)$ and $\left(s \partial_{s}+t \partial_{t}\right) \log D_{n}(s, t)$}

Bear in mind that the auxiliary quantities $R_{n}^{\star}(s, t), R_{n}(s, t), r_{n}^{\star}(s, t)$ and $r_{n}(s, t)$ satisfy four analogs of Riccati equations given by (2.24)-(2.27). We solve for $r_{n}^{\star}(s, t)$ from (2.24) and $r_{n}(s, t)$ from (2.25), and substitute them into (2.26) and (2.27), to establish two second order partial differential equations for $R_{n}^{\star}(s, t)$ and $R_{n}(s, t)$.

Theorem 2.1. The auxiliary quantities $R_{n}^{\star}(s, t)$ and $R_{n}(s, t)$ satisfy a pair of second order nonlinear partial differential equations:

$$
\begin{aligned}
0= & s\left(s \partial_{s s}+t \partial_{s t}\right) R_{n}^{\star}+t\left(s \partial_{s s}+t \partial_{s t}\right) R_{n}+\frac{R_{n}^{\star}-1}{2 R_{n}^{\star}}\left(R_{n}^{(s)}\right)^{2}+\frac{\left(R_{n}^{\star}-1\right) R_{n}^{\star}}{2 R_{n}^{2}}\left(R_{n}^{(t)}\right)^{2} \\
& -\frac{R_{n}^{\star}}{R_{n}} R_{n}^{(s)} R_{n}^{(t)}-t R_{n} R_{n}^{(s)}+t R_{n}^{\star} R_{n}^{(t)}+\left(s \partial_{s}+t \partial_{t}\right) R_{n}^{\star}-s t\left(\partial_{s} R_{n}\right) \\
& -R_{n}^{\star}\left(s R_{n}^{\star}+t R_{n}\right)^{2}+s R_{n}^{\star}\left(s R_{n}^{\star}+t R_{n}\right)-(2 n+1+\alpha) R_{n}^{\star}\left(s R_{n}^{\star}+t R_{n}-s\right) \\
& +\frac{s^{2}}{2} R_{n}^{\star}\left(R_{n}^{\star}-1\right)+\frac{R_{n}^{\star}\left(1-R_{n}^{\star}\right)}{2 R_{n}^{2}}-\alpha \frac{R_{n}^{\star}}{R_{n}},
\end{aligned}
$$

and

$$
\begin{aligned}
0= & s\left(t \partial_{t t}+s \partial_{s t}\right) R_{n}^{\star}+t\left(t \partial_{t t}+s \partial_{s t}\right) R_{n}+\frac{R_{n}}{2 R_{n}^{\star}}\left(R_{n}^{(s)}\right)^{2}+\frac{\left(R_{n}^{\star}-2\right)}{2 R_{n}}\left(R_{n}^{(t)}\right)^{2} \\
& -R_{n}^{(s)} R_{n}^{(t)}+s R_{n} R_{n}^{(s)}-s R_{n}^{\star} R_{n}^{(t)}+\left(s \partial_{s}+t \partial_{t}\right) R_{n}+s^{2}\left(\partial_{t} R_{n}^{\star}\right) \\
& -R_{n}\left(s R_{n}^{\star}+t R_{n}\right)^{2}-(2 n+1+\alpha) R_{n}\left(s R_{n}^{\star}+t R_{n}\right)+\frac{s^{2}}{2} R_{n}^{\star} R_{n}+\frac{2-R_{n}^{\star}}{2 R_{n}}-\alpha .
\end{aligned}
$$

Here,

$$
\begin{aligned}
& R_{n}^{(s)}(s, t):=s \partial_{s} R_{n}^{\star}(s, t)+t \partial_{s} R_{n}(s, t), \\
& R_{n}^{(t)}(s, t):=s \partial_{t} R_{n}^{\star}(s, t)+t \partial_{t} R_{n}(s, t) .
\end{aligned}
$$

Define

$$
H_{n}(s, t):=\left(s \partial_{s}+t \partial_{t}\right) \log D_{n}(s, t),
$$

then it follows from (2.28) and (2.29) that

$$
\begin{aligned}
H_{n}(s, t) & =-\sum_{j=0}^{n-1}\left(s R_{j}^{\star}(s, t)+t R_{j}(s, t)\right) \\
& =p(n, s, t)+n(n+\alpha) .
\end{aligned}
$$

Since $\partial_{s} p(n, s, t)=r_{n}^{\star}(s, t)$ and $\partial_{t} p(n, s, t)=r_{n}(s, t)$ due to (2.21), we find from (2.37)

$$
\begin{aligned}
& \partial_{s} H_{n}(s, t)=r_{n}^{\star}(s, t), \\
& \partial_{t} H_{n}(s, t)=r_{n}(s, t) .
\end{aligned}
$$

In order to characterize the Hankel determinant, we first establish the connection between $H_{n}(s, t)$ and the quantities $R_{n}^{\star}(s, t)$ and $R_{n}(s, t)$, and then make use of (2.35) to derive the partial differential equation satisfied by $H_{n}(s, t)$. 
Shulin Lyu et al. / The Hankel determinant associated with a singularly perturbed Laguerre unitary ensemble

Theorem 2.2. The quantity $H_{n}(s, t)$ is expressible in terms of $R_{n}^{\star}(s, t)$ and $R_{n}(s, t)$ as

$$
\begin{aligned}
H_{n}(s, t)= & -\frac{1}{4 R_{n}^{\star}}\left(R_{n}^{(s)}\right)^{2}+\frac{1-R_{n}^{\star}}{4 R_{n}^{2}}\left(R_{n}^{(t)}\right)^{2}+\frac{R_{n}^{(s)} R_{n}^{(t)}}{2 R_{n}} \\
& -\frac{1}{4}\left(s R_{n}^{\star}+t R_{n}\right)^{2}-\left(n+\frac{\alpha}{2}\right)\left(s R_{n}^{\star}+t R_{n}\right)+\frac{s^{2}}{4} R_{n}^{\star} \\
& -\frac{\alpha^{2}}{4}+\frac{t}{2}+\frac{\alpha}{2 R_{n}}+\frac{R_{n}^{\star}-1}{4 R_{n}^{2}},
\end{aligned}
$$

where $R_{n}^{(s)}(s, t)$ and $R_{n}^{(t)}(s, t)$ have the same meaning as in (2.36). Moreover, $H_{n}(s, t)$ satisfies the following second order sixth degree partial differential equation:

$$
\begin{aligned}
& {\left[\left(\left(t \partial_{t t}+s \partial_{s t}\right) H_{n}\right)^{2}+4\left(\left(\partial_{t} H_{n}\right)-1\right)\left(\partial_{t} H_{n}\right)\left(\left(s \partial_{s}+t \partial_{t}\right) H_{n}-H_{n}+n(n+\alpha)\right)\right]^{2}} \\
& \quad \cdot\left[\left(\left(s \partial_{s s}+t \partial_{s t}\right) H_{n}\right)^{2}+4\left(\partial_{s} H_{n}\right)^{2}\left(\left(s \partial_{s}+t \partial_{t}\right) H_{n}-H_{n}+n(n+\alpha)\right)\right] \\
& =\left[\left(\left(t \partial_{t t}+s \partial_{s t}\right) H_{n}\right)^{2}\left(\left(\partial_{s} H_{n}\right)^{2}+\left(s \partial_{s}+t \partial_{t}\right) H_{n}-H_{n}+n(n+\alpha)\right)\right. \\
& \quad+\left(\left(s \partial_{s s}+t \partial_{s t}\right) H_{n}\right)^{2}\left(\left(\partial_{t} H_{n}\right)-1\right)\left(\partial_{t} H_{n}\right) \\
& \quad-\left(\left(t \partial_{t t}+s \partial_{s t}\right) H_{n}\right)\left(\left(s \partial_{s s}+t \partial_{s t}\right) H_{n}\right)\left((2 n+\alpha)\left(\partial_{t} H_{n}\right)+\left(\partial_{s} H_{n}\right)\left(2\left(\partial_{t} H_{n}\right)-1\right)-n\right) \\
& +\left(\left(s \partial_{s}+t \partial_{t}\right) H_{n}-H_{n}+n(n+\alpha)\right) \\
& \quad \cdot\left(-2\left(2\left(\partial_{t} H_{n}\right)-1\right)\left((2 n+\alpha)\left(\partial_{t} H_{n}\right)-n\right)\left(\partial_{s} H_{n}\right)-\left(n-(2 n+\alpha)\left(\partial_{t} H_{n}\right)\right)^{2}\right. \\
& \left.\left.\quad-\left(\partial_{s} H_{n}\right)^{2}+4\left(\left(\partial_{t} H_{n}\right)-1\right)\left(\partial_{t} H_{n}\right)\left(\left(s \partial_{s}+t \partial_{t}\right) H_{n}-H_{n}+n(n+\alpha)\right)\right)\right]^{2} .
\end{aligned}
$$

Proof. Keeping (2.11) in mind, we get from (2.18) and (2.37)

$$
\begin{aligned}
H_{n}= & s r_{n}^{\star}+t r_{n}-\beta_{n}+n(n+\alpha) \\
= & s r_{n}^{\star}+t r_{n}-\frac{1}{R_{n}}\left(n-(2 n+\alpha) r_{n}-2 r_{n} r_{n}^{\star}+r_{n}^{\star}\right) \\
& +\frac{r_{n}^{2}-r_{n}}{R_{n}^{2}}\left(1-R_{n}^{\star}\right)-\frac{\left(r_{n}^{\star}\right)^{2}}{R_{n}^{\star}}+n(n+\alpha) .
\end{aligned}
$$

Eliminating $r_{n}^{\star}$ and $r_{n}$ from the second equality above by using (2.24) and (2.25) respectively, after simplification, we come to (2.39).

Now that we have the expression for $H_{n}$ in terms of $R_{n}^{\star}$ and $R_{n}$, in order to establish the differential equation for $H_{n}$, it suffices to derive the representations of $R_{n}^{\star}$ and $R_{n}$ in terms of $H_{n}$. To achieve the desired results, we first remove $R_{n-1}^{\star}$ and $R_{n-1}$ from (2.20) by using (2.5) and (2.6), and get

$$
\begin{aligned}
& \partial_{s}\left(\log \beta_{n}\right)=\frac{\left(r_{n}^{\star}\right)^{2}}{\beta_{n} R_{n}^{\star}}-R_{n}^{\star}, \\
& \partial_{t}\left(\log \beta_{n}\right)=\frac{r_{n}^{2}-r_{n}}{\beta_{n} R_{n}}-R_{n} .
\end{aligned}
$$


Solving for $R_{n}^{\star}$ and $R_{n}$ from these two quadratic equations in $R_{n}^{\star}$ and $R_{n}$ respectively, we obtain

$$
\begin{aligned}
& R_{n}^{\star}=-\frac{1}{2} \partial_{s} \log \beta_{n}+\frac{1}{2} \sqrt{\left(\partial_{s} \log \beta_{n}\right)^{2}+\frac{4\left(r_{n}^{\star}\right)^{2}}{\beta_{n}}}, \\
& R_{n}=-\frac{1}{2} \partial_{t} \log \beta_{n}+\frac{1}{2} \sqrt{\left(\partial_{t} \log \beta_{n}\right)^{2}+\frac{4\left(r_{n}^{2}-r_{n}\right)}{\beta_{n}}} .
\end{aligned}
$$

Here take note that there should be two solutions for both $R_{n}^{\star}$ and $R_{n}$, however, the solutions with minus sign before the square roots in (2.41) are rejected, since from (2.20) we see that

$$
\begin{aligned}
R_{n}^{\star}+\frac{1}{2} \partial_{s} \log \beta_{n} & =\frac{1}{2}\left(R_{n-1}^{\star}+R_{n}^{\star}\right) \geq 0, \\
R_{n}+\frac{1}{2} \partial_{t} \log \beta_{n} & =\frac{1}{2}\left(R_{n-1}+R_{n}\right) \geq 0,
\end{aligned}
$$

where we have used the fact that $R_{n}^{\star} \geq 0$ and $R_{n} \geq 0$ which is a direct consequence of their definitions.

From (2.23) and (2.37), we see that

$$
\beta_{n}=\left(s \partial_{s}+t \partial_{t}\right) H_{n}-H_{n}+n(n+\alpha)
$$

Substituting this equation and (2.38) into (2.41), we obtain two equations expressing $R_{n}^{\star}$ and $R_{n}$ in terms of $H_{n}$ and its derivatives. Plugging these expressions into (2.39), we establish (2.40).

Remark 2.1. Suppose $H_{n}$ is independent of $s$, equation (2.40) reduces to

$$
\left(t H_{n}^{\prime \prime}\right)^{2}=\left(n-(2 n+\alpha) H_{n}^{\prime}\right)^{2}-4\left(H_{n}^{\prime}-1\right) H_{n}^{\prime}\left(t H_{n}^{\prime}-H_{n}+n(n+\alpha)\right),
$$

which is consistent with (3.24) in [13]. With a change of variable $t=\tilde{t}^{2}$ and a linear shift

$$
H_{n}(t)=\frac{\sigma(\tilde{t})}{2}+\frac{\tilde{t}^{2}}{2}+\frac{n(n+\alpha)}{2}
$$

we see that $\sigma(\tilde{t})$ satisfies the Jimbo-Miwa-Okamoto $\sigma$-form of Painlevé III [24] with $\theta_{0}=\alpha$ and $\theta_{\infty}=2 n+\alpha$.

Remark 2.2. In case $t=0$, our Hankel determinant $D_{n}(s, t)$ reads

$$
D_{n}(s, 0)=\operatorname{det}\left(\int_{s}^{\infty} x^{i+j} x^{\alpha} \mathrm{e}^{-x} d x\right)_{i, j=0}^{n-1}
$$

which is the special case where $A=0$ and $B=1$ in the Hankel determinant studied in [1]. Our results for $t=0$ are in agreement with the ones produced in [1] where $R_{n}(t)$ and $r_{n}(t)$ correspond to $R_{n}^{\star}(s, 0)$ and $r_{n}^{\star}(s, 0)$ in our paper respectively. 
In fact, when $t=0$, according to the definitions of the auxiliary quantities and through integration by parts, we find

$$
\begin{aligned}
\alpha R_{n}(s, 0) & =1-R_{n}^{\star}(s, 0), \\
\alpha r_{n}(s, 0) & =-n-r_{n}^{\star}(s, 0) .
\end{aligned}
$$

With the aid of these two identities, we set $t=0$ in (2.24) and (2.26) to obtain

$$
\begin{aligned}
s\left(\frac{d}{d s} R_{n}^{\star}(s, 0)\right)= & 2 r_{n}^{\star}(s, 0)+\left(2 n+\alpha-s+s R_{n}^{\star}(s, 0)\right) R_{n}^{\star}(s, 0), \\
s\left(\frac{d}{d s} r_{n}^{\star}(s, 0)\right)= & \frac{\left(1-2 R_{n}^{\star}(s, 0)\right)\left(r_{n}^{\star}(s, 0)\right)^{2}}{R_{n}^{\star}(s, 0)\left(1-R_{n}^{\star}(s, 0)\right)}-(2 n+\alpha) \frac{R_{n}^{\star}(s, 0) r_{n}^{\star}(s, 0)}{1-R_{n}^{\star}(s, 0)} \\
& -n(n+\alpha) \frac{R_{n}^{\star}(s, 0)}{1-R_{n}^{\star}(s, 0)},
\end{aligned}
$$

which is in accord with the Riccati equations (4.5) and (4.6) in [1]. Further, setting $t=0$ in (2.25) and (2.27), in view of (2.42) and (2.43), and taking account of the above two Riccati equations, we find

$$
\begin{gathered}
\left.\alpha\left(\partial_{t} R_{n}^{\star}(s, t)\right)\right|_{t=0}=-\frac{d}{d s} R_{n}^{\star}(s, 0), \\
\left.\alpha\left(\partial_{t} r_{n}^{\star}(s, t)\right)\right|_{t=0}=-\frac{d}{d s} r_{n}^{\star}(s, 0) .
\end{gathered}
$$

We differentiate (2.44) with respect to $s$ and get

$$
\left.\alpha\left(\partial_{s t} R_{n}^{\star}(s, t)\right)\right|_{t=0}=-\frac{d^{2}}{d s^{2}} R_{n}^{\star}(s, 0) .
$$

Substituting (2.42), (2.44) and (2.46) into (2.35a) with $t=0$, we establish an ordinary differential equation satisfied by $R_{n}^{\star}(s, 0)$, which is consistent with the result given by Theorem 5 in [1].

According to (2.38), i.e. $\partial_{s} H_{n}(s, t)=r_{n}^{\star}(s, t), \partial_{t} H_{n}(s, t)=r_{n}(s, t)$, and by using (2.43) and (2.45), we come to

$$
\begin{gathered}
\left.\alpha\left(\partial_{t} H_{n}(s, t)\right)\right|_{t=0}=-n-\frac{d}{d s} H_{n}(s, 0), \\
\left.\alpha\left(\partial_{t s} H_{n}(s, t)\right)\right|_{t=0}=-\frac{d^{2}}{d s^{2}} H_{n}(s, 0) .
\end{gathered}
$$

Setting $t=0$ in (2.40) and plugging the above two equalities into it, we produce an ordinary differential equation satisfied by $H_{n}(s, 0)=s \frac{d}{d s} \log D_{n}(s, 0)$, which agrees with equation (4.11) in [1].

\section{Double Scaling for Large $\boldsymbol{n}$ and Asymptotic Expansions for the Scaled Hankel Determinant}

Based on the large partial differential equations satisfied by $R_{n}^{\star}(s, t), R_{n}(s, t)$ and $H_{n}(s, t)$, we proceed with the study of the Hankel determinant as $n$ gets large, under the assumption that $n \rightarrow \infty, s \rightarrow 0$ and $t \rightarrow 0$ such that $S$ and $T$ defined by

$$
S:=4 n s, \quad T:=(2 n+1+\alpha) t,
$$

are fixed. This scaling is motivated as follows: for $t=0$ and under the scaling $S=4 n s$, the limiting behavior of the smallest eigenvalue distribution of Laguerre unitary ensemble is characterized by 
the Bessel kernel [33]; for $s=0$ and under the scaling $T=(2 n+1+\alpha) t$, the eigenvalue correlation kernel of the singularly perturbed Laguerre unitary ensemble is given by the $\Psi$-kernel [34, Corollary $1]$.

To proceed further, we make the Ansatz that $R_{n}^{\star}(s, t), R_{n}(s, t)$, and their first and second order partial derivatives over $s$ or $t$, have limits as $n \rightarrow \infty$. Define

$$
\begin{aligned}
2 R^{\star}(S, T) & :=\lim _{n \rightarrow \infty} R_{n}^{\star}(s, t), \\
R(S, T) & :=\lim _{n \rightarrow \infty} R_{n}(s, t) .
\end{aligned}
$$

Then, on replacing $s$ by $\frac{S}{4 n}$ and $t$ by $\frac{T}{2 n+1+\alpha}$ in (2.24) and (2.25), and by sending $n$ to $\infty$, we find

$$
\begin{aligned}
& \lim _{n \rightarrow \infty} \frac{r_{n}^{\star}(s, t)}{n}=-2 R^{\star}(S, T), \\
& \lim _{n \rightarrow \infty} \frac{r_{n}(s, t)}{n}=-R(S, T) .
\end{aligned}
$$

Moreover, we derive the following limiting equations from (2.35).

Theorem 3.1. The quantities $R^{\star}(S, T)$ and $R(S, T)$ satisfy a couple of second order first degree partial differential equations

$$
\begin{aligned}
0= & S\left(S \partial_{S S}+T \partial_{S T}\right) R^{\star}+T\left(S \partial_{S S}+T \partial_{S T}\right) R \\
& +\frac{2 R^{\star}-1}{2 R^{\star}}\left(S\left(\partial_{S} R^{\star}\right)+T\left(\partial_{S} R\right)\right)^{2}+\frac{\left(2 R^{\star}-1\right) R^{\star}}{2 R^{2}}\left(S\left(\partial_{T} R^{\star}\right)+T\left(\partial_{T} R\right)\right)^{2} \\
& -\frac{2 R^{\star}}{R}\left(S\left(\partial_{S} R^{\star}\right)+T\left(\partial_{S} R\right)\right)\left(S\left(\partial_{T} R^{\star}\right)+T\left(\partial_{T} R\right)\right)+\left(S \partial_{S}+T \partial_{T}\right) R^{\star} \\
& -R^{\star}\left(S R^{\star}+T R\right)+\frac{S}{2} R^{\star}+\frac{R^{\star}\left(1-2 R^{\star}\right)}{2 R^{2}}-\alpha \frac{R^{\star}}{R},
\end{aligned}
$$

and

$$
\begin{aligned}
0= & S\left(T \partial_{T T}+S \partial_{T S}\right) R^{\star}+T\left(T \partial_{T T}+S \partial_{T S}\right) R \\
& +\frac{R}{R^{\star}}\left(S\left(\partial_{S} R^{\star}\right)+T\left(\partial_{S} R\right)\right)^{2}+\frac{\left(R^{\star}-1\right)}{R}\left(S\left(\partial_{T} R^{\star}\right)+T\left(\partial_{T} R\right)\right)^{2} \\
& -2\left(S\left(\partial_{S} R^{\star}\right)+T\left(\partial_{S} R\right)\right)\left(S\left(\partial_{T} R^{\star}\right)+T\left(\partial_{T} R\right)\right)+\left(S \partial_{S}+T \partial_{T}\right) R \\
& -R\left(S R^{\star}+T R\right)+\frac{1-R^{\star}}{R}-\alpha .
\end{aligned}
$$

Proof. Adopting ideas in [30], we proceed as follows.

From (3.1), i.e. $R_{n}^{\star}(s, t) \sim 2 R^{\star}(s, t), R_{n}(s, t) \sim R(S, T)$ as $n \rightarrow \infty$, it follows that

$$
\begin{aligned}
& \partial_{S} R_{n}^{\star} \sim 2 \partial_{S} R^{\star} \cdot 4 n, \\
& \partial_{s} R_{n} \sim \partial_{S} R \cdot 4 n, \\
& \partial_{t} R_{n}^{\star} \sim 2 \partial_{T} R^{\star} \cdot(2 n+1+\alpha), \\
& \partial_{t} R_{n} \sim \partial_{T} R \cdot(2 n+1+\alpha), \\
& \partial_{s t} R_{n}^{\star} \sim 2 \partial_{S T} R^{\star} \cdot 4 n \cdot(2 n+1+\alpha), \\
& \partial_{s t} R_{n} \sim \partial_{S T} R \cdot 4 n \cdot(2 n+1+\alpha), \\
& \partial_{s s} R_{n}^{\star} \sim 2 \partial_{S S} R^{\star} \cdot(4 n)^{2}, \\
& \partial_{s s} R_{n} \sim \partial_{S S} R \cdot(4 n)^{2}, \\
& \partial_{t t} R_{n}^{\star} \sim 2 \partial_{T T} R^{\star} \cdot(2 n+1+\alpha)^{2}, \\
& \partial_{t t} R_{n} \sim \partial_{T T} R \cdot(2 n+1+\alpha)^{2} .
\end{aligned}
$$


Replacing the first and second order partial derivatives of $R_{n}^{\star}(s, t)$ and $R_{n}(s, t)$ in (2.35) by using the above relations, and substituting $2 R^{\star}, R, \frac{S}{4 n}, \frac{T}{2 n+1+\alpha}$ for $R_{n}^{\star}, R_{n}, s, t$ respectively, by taking the series expansion in $n$ of the resulting equation and with the aid of the software Mathematica, we obtain (3.3) by retaining leading order terms when $n \rightarrow \infty$.

The next proposition gives us the lower and upper bounds for $R^{\star}(S, T)$ and $R(S, T)$, which result from the definitions of $R_{n}^{\star}(s, t)$ and $R_{n}(s, t)$.

Proposition 3.1. For $\alpha>0$, the auxiliary quantities $R_{n}^{\star}(s, t)$ and $R_{n}(s, t)$ are bounded by

$$
\begin{aligned}
& 0 \leq R_{n}^{\star}(s, t)<1, \\
& 0<R_{n}(s, t) \leq \frac{1}{\alpha},
\end{aligned}
$$

with the lower bound for $R_{n}^{\star}(s, t)$ and the upper bound for $R_{n}(s, t)$ attained only for $s=0$ and $s=t=0$ respectively. As a consequence, we have for $\alpha>0$,

$$
\begin{aligned}
& 0 \leq R^{\star}(S, T) \leq \frac{1}{2}, \\
& 0 \leq R(S, T) \leq \frac{1}{\alpha} .
\end{aligned}
$$

Proof. Recall that

$$
\begin{aligned}
& R_{n}^{\star}(s, t)=\frac{w(s ; t)}{h_{n}(s, t)} P_{n}^{2}(s ; s, t) \geq 0, \\
& R_{n}(s, t)=\frac{1}{h_{n}(s, t)} \int_{s}^{\infty} \frac{d y}{y} P_{n}^{2}(y ; s, t) w(y ; t)>0 .
\end{aligned}
$$

Through integration by parts, we find

$$
\begin{aligned}
0<\alpha R_{n}(s, t) & =\frac{1}{h_{n}(s, t)} \int_{s}^{\infty} P_{n}^{2}(x ; s, t) \mathrm{e}^{-x-\frac{t}{x}} d x^{\alpha} \\
& =1-R_{n}^{\star}(s, t)-\frac{t}{h_{n}(s, t)} \int_{s}^{\infty} \frac{d y}{y^{2}} P_{n}^{2}(y ; s, t) w(y ; t) \\
& \leq 1-R_{n}^{\star}(s, t) \leq 1 .
\end{aligned}
$$

Hence, we have

$$
0<\alpha R_{n}(s, t) \leq 1-R_{n}^{\star}(s, t) \leq 1,
$$

where the first and second equality sign hold if and only if $t=0$ and $s=0$ respectively. Therefore, we see that

$$
0 \leq R_{n}^{\star}(s, t)<1 \quad \text { and } \quad 0<R_{n}(s, t) \leq \frac{1}{\alpha},
$$

where the equality signs are valid only for $s=0$ and $s=t=0$ respectively. Finally, by using the property of the limit of a function, we obtain

$$
\begin{gathered}
0 \leq R^{\star}(S, T)=\frac{1}{2} \lim _{n \rightarrow \infty} R_{n}^{\star}(s, t) \leq \frac{1}{2}, \\
0 \leq R(S, T)=\lim _{n \rightarrow \infty} R_{n}(s, t) \leq \frac{1}{\alpha} .
\end{gathered}
$$


To continue, for $s=S /(4 n)$ and $t=T /(2 n+1+\alpha)$, we let

$$
\begin{aligned}
H(S, T): & =\lim _{n \rightarrow \infty} H_{n}(s, t) \\
& =\left(S \partial_{S}+T \partial_{T}\right) \log \Delta(S, T) .
\end{aligned}
$$

Here recall that $H_{n}(s, t):=\left(s \partial_{s}+t \partial_{t}\right) D_{n}(s, t)$ and $\Delta(S, T)$ is defined by

$$
\Delta(S, T):=\lim _{n \rightarrow \infty} \frac{D_{n}\left(\frac{S}{4 n}, \frac{T}{2 n+1+\alpha}\right)}{D_{n}(0,0)} .
$$

It follows from (2.38) and (3.2) that

$$
\begin{aligned}
& \partial_{S} H(S, T)=\lim _{n \rightarrow \infty} \frac{r_{n}^{\star}(s, t)}{4 n}=-\frac{1}{2} R^{\star}(S, T), \\
& \partial_{T} H(S, T)=\lim _{n \rightarrow \infty} \frac{r_{n}(s, t)}{2 n+1+\alpha}=-\frac{1}{2} R(S, T) .
\end{aligned}
$$

Furthermore, by an argument similar to the one used in the proof of Theorem 3.1, we establish the following statement from (2.39) and (2.40).

Theorem 3.2. The quantity $H(S, T)$ is expressed in terms of $R^{\star}(S, T)$ and $R(S, T)$ by

$$
\begin{aligned}
H(S, T)= & -\frac{1}{2 R^{\star}}\left(S\left(\partial_{S} R^{\star}\right)+T\left(\partial_{S} R\right)\right)^{2}+\frac{1-2 R^{\star}}{4 R^{2}}\left(S\left(\partial_{T} R^{\star}\right)+T\left(\partial_{T} R\right)\right)^{2} \\
& +\frac{1}{R}\left(S\left(\partial_{S} R^{\star}\right)+T\left(\partial_{S} R\right)\right)\left(S\left(\partial_{T} R^{\star}\right)+T\left(\partial_{T} R\right)\right) \\
& -\frac{1}{2}\left(S R^{\star}+T R\right)-\frac{\alpha^{2}}{4}+\frac{\alpha}{2 R}+\frac{2 R^{\star}-1}{4 R^{2}},
\end{aligned}
$$

and the following second order second degree partial differential equation holds:

$$
\begin{aligned}
0= & 4 \frac{\left(\partial_{T} H\right)^{2}}{\partial_{S} H}\left(\left(S \partial_{S S}+T \partial_{S T}\right) H\right)^{2}+\left(4\left(\partial_{S} H\right)+1\right)\left(\left(T \partial_{T T}+S \partial_{T S}\right) H\right)^{2} \\
& -8\left(\partial_{T} H\right)\left(\left(S \partial_{S S}+T \partial_{S T}\right) H\right)\left(\left(T \partial_{T T}+S \partial_{T S}\right) H\right) \\
& -\left(\alpha\left(\partial_{T} H\right)+\frac{1}{2}\right)^{2}+4\left(\left(S \partial_{S}+T \partial_{T}-1\right) H\right)\left(\partial_{T} H\right)^{2}-\partial_{S} H
\end{aligned}
$$

Remark 3.1. In case $S=0$, equations (3.3)-(3.6) coincide with the corresponding ones produced in [5] where $s, C(s)$ and $\mathscr{H}(s)$ correspond to $T, R(0, T)$ and $H(0, T)$ in our paper respectively.

For $T=0$ or $t=0$, we reproduce (3.7), (3.9) and (3.10) of [27] by making use of (3.3a), (3.5) and (3.6). Note that our $S, R^{\star}(S, 0)$ and $H(S, 0)$ corresponds to $s, \frac{1}{2} R(s)$ and $\sigma(s)$ of [27] respectively.

Before proceeding with the derivation the asymptotic expansions of $R^{\star}(S, T), R(S, T)$ and $H(S, T)$ by using (3.3)-(3.6), we first make an important observation. On dropping the terms with derivatives in (3.3) and (3.5), and by using $\widetilde{R}^{\star}(S, T), \widetilde{R}(S, T)$ and $\widetilde{H}(S, T)$ in place of $R^{\star}(S, T)$, 
$R(S, T)$ and $H(S, T)$ respectively, we get

$$
\begin{aligned}
&-\widetilde{R}^{\star}\left(S \widetilde{R}^{\star}+T \widetilde{R}\right)+ \frac{S}{2} \widetilde{R}^{\star}+\frac{\widetilde{R}^{\star}\left(1-2 \widetilde{R}^{\star}\right)}{2(\widetilde{R})^{2}}-\alpha \frac{\widetilde{R}^{\star}}{\widetilde{R}}=0, \\
&-\widetilde{R}\left(S \widetilde{R}^{\star}+T \widetilde{R}\right)+\frac{1-\widetilde{R}^{\star}}{\widetilde{R}}-\alpha=0,
\end{aligned}
$$

and

$$
\widetilde{H}(S, T)=-\frac{1}{2}\left(S \widetilde{R}^{\star}+T \widetilde{R}\right)-\frac{\alpha^{2}}{4}+\frac{\alpha}{2 \widetilde{R}}+\frac{2 \widetilde{R}^{\star}-1}{4(\widetilde{R})^{2}}
$$

Solving for $\widetilde{R}^{\star}(S, T)$ and $\widetilde{R}(S, T)$ from (3.7) yields

$$
\widetilde{R}^{\star}(S, T)=\frac{1}{2}-\frac{\alpha}{2 \sqrt{S}}-\frac{T}{2 S^{\frac{3}{2}}}, \quad \widetilde{R}(S, T)=\frac{1}{\sqrt{S}},
$$

where we have used the assumption that $\widetilde{R}>0$. Hence, from (3.8), it follows that

$$
\widetilde{H}(S, T)=-\frac{S}{4}+\frac{\alpha}{2} \sqrt{S}-\frac{\alpha^{2}}{4}-\frac{T}{2 \sqrt{S}} .
$$

From (3.9), we are able to guess a "reasonable" form and the leading terms of the asymptotic expansion of

$$
H(S, T)=\left(S \partial_{S}+T \partial_{T}\right) \log \Delta(S, T, \alpha)
$$

Using the complete expansion of $H(S, T)$ derived from (3.6), after integration and exponentiating, we establish the asymptotic expansion of the scaled Hankel determinant

$$
\Delta(S, T, \alpha)=\lim _{n \rightarrow \infty} \frac{D_{n}\left(\frac{S}{4 n}, \frac{T}{2 n+1+\alpha}, \alpha\right)}{D_{n}(0,0, \alpha)} .
$$

Note that here and in the rest of this paper, we will not display $\alpha$-dependence of the double-scaled objects, to ease notations.

In addition to this, by making use of the following relations given by (3.4):

$$
R^{\star}(S, T)=-2 \partial_{S} H(S, T), \quad R(S, T)=-2 \partial_{T} H(S, T),
$$

we can easily deduce the asymptotic expansions of $R^{\star}(S, T)$ and $R(S, T)$ from the one of $H(S, T)$. 
Shulin Lyu et al. / The Hankel determinant associated with a singularly perturbed Laguerre unitary ensemble

\subsection{Asymptotic expansions for large $S$ and fixed $T$}

We assume $H(S, T)$ with $T$ fixed has an expansion for large $S$ of the following form

$$
H(S, T)=\sum_{j=0}^{\infty} a_{j}(T) S^{1-\frac{j}{2}}, \quad S \rightarrow \infty
$$

As is pointed out in Remark 3.1, the special case $H(S, 0)$ is investigated in [27] where the symbol $\sigma(s)$ was used instead. According to (3.13) in [27], $H(S, 0)$ has the following expansion

$$
\begin{aligned}
H(S, 0)= & -\frac{S}{4}+\frac{\alpha}{2} \sqrt{S}-\frac{\alpha^{2}}{4}-\frac{\alpha}{16 \sqrt{S}}-\frac{\alpha^{2}}{16 S}-\left(\frac{\alpha^{3}}{16}+\frac{9 \alpha}{256}\right) S^{-\frac{3}{2}} \\
& -\left(\frac{\alpha^{4}}{16}+\frac{9 \alpha^{2}}{64}\right) S^{-2}-\left(\frac{\alpha^{5}}{16}+\frac{45 \alpha^{3}}{128}+\frac{225 \alpha}{2048}\right) S^{-\frac{5}{2}} \\
& -\left(\frac{\alpha^{6}}{16}+\frac{45 \alpha^{4}}{64}+\frac{27 \alpha^{2}}{32}\right) S^{-3}+O\left(S^{-\frac{7}{2}}\right) \\
= & \sum_{j=0}^{\infty} a_{j}(0) S^{1-\frac{j}{2}}, \quad S \rightarrow \infty
\end{aligned}
$$

which provides initial conditions satisfied by $a_{j}(T)$ at $T=0$. For example, we have $a_{0}(0)=$ $-\frac{1}{4}, a_{1}(0)=\frac{\alpha}{2}$ and $a_{2}(0)=-\frac{\alpha^{2}}{4}$.

Substituting (3.10) into (3.6), on comparing the coefficients of the highest order term in $S$ on both sides, we get $a_{0}(T) a_{0}^{\prime}(T)=0$ which indicates that $a_{0}(T)$ must be a constant. Hence we have $a_{0}(T)=a_{0}(0)=-\frac{1}{4}$. Successive coefficients $a_{j}(T), j=1,2, \cdots$, are computed similarly, and we finally arrive at

$$
\begin{aligned}
H(S, T)= & -\frac{S}{4}+\frac{\alpha}{2} \sqrt{S}-\frac{\alpha^{2}}{4}-\left(\frac{T}{2}+\frac{\alpha}{16}\right) \frac{1}{\sqrt{S}}-\frac{\alpha^{2}}{16 S} \\
& -\left(\frac{T}{16}+\frac{\alpha^{3}}{16}+\frac{9 \alpha}{256}\right) S^{-\frac{3}{2}}-\left(\frac{\alpha}{8} T+\frac{\alpha^{4}}{16}+\frac{9 \alpha^{2}}{64}\right) S^{-2} \\
& -\left(\left(\frac{3 \alpha^{2}}{16}+\frac{27}{256}\right) T+\frac{\alpha^{5}}{16}+\frac{45 \alpha^{3}}{128}+\frac{225 \alpha}{2048}\right) S^{-\frac{5}{2}} \\
& -\left(\frac{T^{2}}{16}+\left(\frac{\alpha^{3}}{4}+\frac{9 \alpha}{16}\right) T+\frac{\alpha^{6}}{16}+\frac{45 \alpha^{4}}{64}+\frac{27 \alpha^{2}}{32}\right) S^{-3} \\
& +O\left(S^{-\frac{7}{2}}\right), \quad S \rightarrow \infty .
\end{aligned}
$$


Hence, we find

$$
\begin{aligned}
R^{\star}(S, T)= & -2 \partial_{S} H(S, T) \\
= & \frac{1}{2}-\frac{\alpha}{2 \sqrt{S}}-\left(\frac{T}{2}+\frac{\alpha}{16}\right) S^{-\frac{3}{2}}-\frac{\alpha^{2}}{8 S^{2}} \\
& -\left(\frac{3 T}{16}+\frac{3 \alpha^{3}}{16}+\frac{27 \alpha}{256}\right) S^{-\frac{5}{2}}-\left(\frac{\alpha}{2} T+\frac{\alpha^{4}}{4}+\frac{9 \alpha^{2}}{16}\right) S^{-3} \\
& -\left(\left(\frac{15 \alpha^{2}}{16}+\frac{135}{256}\right) T+\frac{5 \alpha^{5}}{16}+\frac{225 \alpha^{3}}{128}+\frac{1125 \alpha}{2048}\right) S^{-\frac{7}{2}} \\
& -\left(\frac{3 T^{2}}{8}+\left(\frac{3 \alpha^{3}}{2}+\frac{27 \alpha}{8}\right) T+\frac{3 \alpha^{6}}{8}+\frac{135 \alpha^{4}}{32}+\frac{81 \alpha^{2}}{16}\right) S^{-4} \\
& +O\left(S^{-\frac{9}{2}}\right), \quad S \rightarrow \infty,
\end{aligned}
$$

and

$$
\begin{aligned}
R(S, T)= & -2 \partial_{T} H(S, T) \\
= & \frac{1}{\sqrt{S}}+\frac{1}{8 S^{\frac{3}{2}}}+\frac{\alpha}{4 S^{2}}+\left(\frac{3 \alpha^{2}}{8}+\frac{27}{128}\right) S^{-\frac{5}{2}} \\
& +\left(\frac{T}{4}+\frac{\alpha^{3}}{2}+\frac{9 \alpha}{8}\right) S^{-3}+O\left(S^{-\frac{7}{2}}\right), \quad S \rightarrow \infty .
\end{aligned}
$$

Since $R^{\star}(S, 0)$ corresponds to $\frac{1}{2} R(s)$ in [27], we remark that our expansion formula for $R^{\star}(S, 0)$ agrees with the one for $R(s)$ (equation (3.12), [27]). ${ }^{\text {a }}$

Noting that $H(S, T)=\left(S \partial_{S}+T \partial_{T}\right) \log \Delta(S, T, \alpha)$, by integration of (3.11), we obtain the following asymptotic expansion of $\Delta(S, T, \alpha)$.

Theorem 3.3. We have, for large $S$ and fixed $T$,

$$
\begin{aligned}
\log \Delta(S, T, \alpha)= & C_{1}\left(\frac{T}{S}, \alpha\right)-\frac{S}{4}+\alpha \sqrt{S}-\frac{\alpha^{2}}{4} \log S \\
& +\left(\frac{\alpha}{8}-T\right) \frac{1}{\sqrt{S}}+\frac{\alpha^{2}}{16 S}+\left(\frac{\alpha^{3}}{24}+\frac{3 \alpha}{128}+\frac{T}{8}\right) S^{-\frac{3}{2}} \\
& +\left(\frac{\alpha^{4}}{32}+\frac{9 \alpha^{2}}{128}+\frac{\alpha}{8} T\right) S^{-2}+O\left(S^{-\frac{5}{2}}\right)
\end{aligned}
$$

where $C_{1}\left(\frac{T}{S}, \alpha\right)$ is an arbitrary function of $\frac{T}{S}$ and $\alpha$.

\subsection{Asymptotic expansions for small $S$ and fixed $T>0$}

Motivated by (3.9), we write for fixed $T>0$,

$$
H(S, T)=-\frac{T}{2 \sqrt{S}}+\sum_{j=0}^{\infty} b_{j}(T) S^{\frac{j}{2}}, \quad S \rightarrow 0 .
$$

${ }^{a}$ The authors note that it would be of interest to perform some numerical analysis to verify the validity of the expansion and the size of the error terms for the expansion (3.11) and those that follow in this section. 
Shulin Lyu et al. / The Hankel determinant associated with a singularly perturbed Laguerre unitary ensemble

Substituting it into (3.6), by comparing the coefficients on both sides, we come to

$$
\begin{aligned}
H(S, T)= & -\frac{T}{2 \sqrt{S}}+\frac{1}{16}-\frac{\alpha^{2}}{4}+\frac{\alpha}{2} \sqrt{S}-\frac{S}{4}+\frac{S^{\frac{3}{2}}}{16 T} \\
& -\frac{\alpha S^{\frac{5}{2}}}{16 T^{2}}+\frac{S^{3}}{16 T^{2}}+\left(\frac{\alpha^{2}}{16}-\frac{27}{256}\right) \frac{S^{\frac{7}{2}}}{T^{3}}-\frac{\alpha S^{4}}{8 T^{3}} \\
& +\left(-\frac{\alpha^{3}}{16}+\frac{99 \alpha}{256}+\frac{T}{16}\right) \frac{S^{\frac{9}{2}}}{T^{4}}+\left(\frac{\alpha^{2}}{16}-\frac{9}{64}\right) \frac{3 S^{5}}{T^{4}} \\
& +O\left(S^{\frac{11}{2}}\right), \quad S \rightarrow 0 .
\end{aligned}
$$

It follows that

$$
\begin{aligned}
R^{\star}(S, T)= & -2 \partial_{S} H(S, T) \\
= & -\frac{T}{2 S^{\frac{3}{2}}}-\frac{\alpha}{2 \sqrt{S}}+\frac{1}{2}-\frac{3 \sqrt{S}}{16 T}+\frac{5 \alpha S^{\frac{3}{2}}}{16 T^{2}}-\frac{3 S^{2}}{8 T^{2}} \\
& -\left(\frac{\alpha^{2}}{16}-\frac{27}{256}\right) \frac{7 S^{\frac{5}{2}}}{T^{3}}+\frac{\alpha S^{3}}{T^{3}}-\left(-\frac{\alpha^{3}}{16}+\frac{99 \alpha}{256}+\frac{T}{16}\right) \frac{9 S^{\frac{7}{2}}}{T^{4}} \\
& -\left(\frac{\alpha^{2}}{8}-\frac{9}{32}\right) \frac{15 S^{4}}{T^{4}}+O\left(S^{\frac{9}{2}}\right), \quad S \rightarrow 0,
\end{aligned}
$$

and

$$
\begin{aligned}
R(S, T)= & -2 \partial_{T} H(S, T) \\
= & \frac{1}{\sqrt{S}}+\frac{S^{\frac{3}{2}}}{8 T^{2}}-\frac{\alpha S^{\frac{5}{2}}}{4 T^{3}}+\frac{S^{3}}{4 T^{3}}+\left(\frac{\alpha^{2}}{8}-\frac{27}{128}\right) \frac{3 S^{\frac{7}{2}}}{T^{4}} \\
& -\frac{3 \alpha S^{4}}{4 T^{4}}+\left(-\frac{\alpha^{3}}{2}+\frac{99 \alpha}{32}+\frac{3 T}{8}\right) \frac{S^{\frac{9}{2}}}{T^{5}} \\
& +\left(\frac{\alpha^{2}}{2}-\frac{9}{8}\right) \frac{3 S^{5}}{T^{5}}+O\left(S^{\frac{11}{2}}\right), \quad S \rightarrow 0 .
\end{aligned}
$$

Since $H(S, T)=\left(S \partial_{S}+T \partial_{T}\right)(\log \Delta(S, T, \alpha))$, we establish the asymptotic expansion of $\Delta(S, T, \alpha)$ by integrating of (3.13).

Theorem 3.4. For small $S$ and fixed $T>0$, we have

$$
\begin{aligned}
\log \Delta(S, T, \alpha)= & C_{2}\left(\frac{T}{S}, \alpha\right)-\frac{T}{\sqrt{S}}+\left(\frac{1}{16}-\frac{\alpha^{2}}{4}\right) \log S \\
& +\alpha \sqrt{S}-\frac{S}{4}+\frac{S^{\frac{3}{2}}}{8 T}-\frac{\alpha S^{\frac{5}{2}}}{8 T^{2}}+\frac{S^{3}}{16 T^{2}} \\
& +\left(\frac{\alpha^{2}}{8}-\frac{27}{128}\right) \frac{S^{\frac{7}{2}}}{T^{3}}-\frac{\alpha S^{4}}{8 T^{3}}+O\left(S^{\frac{9}{2}}\right),
\end{aligned}
$$

where $C_{2}\left(\frac{T}{S}, \alpha\right)$ is an arbitrary function of $\frac{T}{S}$ and $\alpha$. 
Shulin Lyu et al. / The Hankel determinant associated with a singularly perturbed Laguerre unitary ensemble

\subsection{Asymptotic expansions for fixed $S>0$ and large $T$}

According to (3.9), we suppose $H(S, T)$ with $S>0$ fixed has the expansion for large $T$ of the following form

$$
H(S, T)=-\frac{T}{2 \sqrt{S}}+\sum_{j=0}^{\infty} c_{j}(S) T^{-j}, \quad T \rightarrow \infty
$$

Substituting it into (3.6) and comparing the coefficients on both sides, we obtain

$$
\begin{aligned}
H(S, T)= & -\frac{T}{2 \sqrt{S}}-\frac{1}{4}(\sqrt{S}-\alpha)^{2}+\frac{1}{16}+\frac{S^{\frac{3}{2}}}{16 T} \\
& +\frac{S^{\frac{5}{2}}}{16 T^{2}}(\sqrt{S}-\alpha)+\frac{S^{\frac{7}{2}}}{16 T^{3}}\left((\sqrt{S}-\alpha)^{2}-\frac{27}{16}\right) \\
& +\frac{S^{\frac{9}{2}}}{16 T^{4}}\left((\sqrt{S}-\alpha)^{3}-\frac{27}{4} \sqrt{S}+\frac{99 \alpha}{16}\right) \\
& +\frac{S^{\frac{11}{2}}}{16 T^{5}}\left((\sqrt{S}-\alpha)^{4}-\frac{9}{8}(\sqrt{S}-\alpha)(15 \sqrt{S}-13 \alpha)+\frac{2277}{128}\right) \\
& +O\left(T^{-6}\right), \quad T \rightarrow \infty .
\end{aligned}
$$

Therefore, we have

$$
\begin{aligned}
R^{\star}(S, T)= & -2 \partial_{S} H(S, T) \\
= & -\frac{T}{2 S^{\frac{3}{2}}}+\frac{1}{2}-\frac{\alpha}{2 \sqrt{S}}-\frac{3 \sqrt{S}}{16 T}-\frac{S^{\frac{3}{2}}}{16 T^{2}}(6 \sqrt{S}-5 \alpha) \\
& -\frac{S^{\frac{5}{2}}}{16 T^{3}}\left((\sqrt{S}-\alpha)(9 \sqrt{S}-7 \alpha)-\frac{189}{16}\right) \\
& -\frac{S^{\frac{7}{2}}}{16 T^{4}}\left(3(\sqrt{S}-\alpha)^{2}(4 \sqrt{S}-3 \alpha)-\frac{135}{2} \sqrt{S}+\frac{891}{16} \alpha\right) \\
& +O\left(T^{-5}\right), \quad T \rightarrow \infty,
\end{aligned}
$$

and

$$
\begin{aligned}
R(S, T)= & -2 \partial_{T} H(S, T) \\
= & \frac{1}{\sqrt{S}}+\frac{S^{\frac{3}{2}}}{8 T^{2}}+\frac{S^{\frac{5}{2}}}{4 T^{3}}(\sqrt{S}-\alpha)+\frac{3 S^{\frac{7}{2}}}{8 T^{4}}\left((\sqrt{S}-\alpha)^{2}-\frac{27}{16}\right) \\
& +\frac{S^{\frac{9}{2}}}{2 T^{5}}\left((\sqrt{S}-\alpha)^{3}-\frac{27}{4} \sqrt{S}+\frac{99}{16} \alpha\right) \\
& +\frac{5 S^{\frac{11}{2}}}{8 T^{6}}\left((\sqrt{S}-\alpha)^{4}-\frac{9}{8}(\sqrt{S}-\alpha)(15 \sqrt{S}-13 \alpha)+\frac{2277}{128}\right) \\
& +O\left(T^{-7}\right), \quad T \rightarrow \infty .
\end{aligned}
$$

Using the fact that $H(S, T)=\left(S \partial_{S}+T \partial_{T}\right) \log \Delta(S, T, \alpha)$ again, after integration of (3.15), we obtain the asymptotic expansion of $\Delta(S, T, \alpha)$. 
Theorem 3.5. We have, for large $T$ and fixed $S>0$,

$$
\begin{aligned}
\log \Delta(S, T, \alpha)= & C_{3}\left(\frac{T}{S}, \alpha\right)-\frac{T}{\sqrt{S}}-\frac{S}{4}+\alpha \sqrt{S}+\left(\frac{1}{16}-\frac{\alpha^{2}}{4}\right) \log S \\
& +\frac{S^{\frac{3}{2}}}{8 T}+\frac{S^{3}-2 \alpha S^{\frac{5}{2}}}{16 T^{2}}+\frac{S^{\frac{7}{2}}}{24 T^{3}}\left(S-3 \alpha \sqrt{S}+3 \alpha^{2}-\frac{81}{16}\right) \\
& +\frac{S^{\frac{9}{2}}}{32 T^{4}}\left(S^{\frac{3}{2}}-4 \alpha S+\left(6 \alpha^{2}-\frac{27}{2}\right) \sqrt{S}-4 \alpha^{3}+\frac{99}{4} \alpha\right) \\
& +O\left(T^{-5}\right) .
\end{aligned}
$$

Here $C_{3}\left(\frac{T}{S}, \alpha\right)$ is an arbitrary function of $\frac{T}{S}$ and $\alpha$.

To summarize, we have established the asymptotic expansions of the scaled Hankel determinant $D_{n}\left(\frac{S}{4 n}, \frac{T}{2 n+1+\alpha}, \alpha\right), n \rightarrow \infty$, for large $S$ with $T \geq 0$ fixed, for small $S$ with $T>0$ fixed, and for large $T$ with $S>0$ fixed. In each case, there is a function term $C_{j}\left(\frac{T}{S}, \alpha\right)$ to be determined. In order to compute them, we turn our attention back to the monic polynomials $P_{n}(x ; s, t, \alpha)$ orthogonal with respect to $w(x ; t, \alpha)=x^{\alpha} \mathrm{e}^{-x-\frac{t}{x}}$ over $[s, \infty)$ with $\alpha>-1, t \geq 0, s \geq 0$.

We have the following determinant representation for $P_{n}(z ; s, t, \alpha)$ in terms of the moments (see Szegö [32], Chapter II)

$$
P_{n}(z ; s, t, \alpha)=\frac{1}{D_{n}(s, t, \alpha)} \operatorname{det}\left(\int_{s}^{\infty} x^{i+j}(z-x) x^{\alpha} \mathrm{e}^{-x-\frac{t}{x}} d x\right)_{i, j=0}^{n-1}
$$

Keeping in mind that $D_{n}(s, t, \alpha)$ is the Hankel determinant generated by the moments of $w(x ; t, \alpha)$, i.e.

$$
D_{n}(s, t, \alpha)=\operatorname{det}\left(\int_{s}^{\infty} x^{i+j} x^{\alpha} \mathrm{e}^{-x-\frac{t}{x}} d x\right)_{i, j=0}^{n-1},
$$

we readily see from (3.17) that

$$
P_{n}(0 ; s, t, \alpha)=(-1)^{n} \frac{D_{n}(s, t, \alpha+1)}{D_{n}(s, t, \alpha)} .
$$

Hence, by recalling that

$$
\Delta(S, T, \alpha)=\lim _{n \rightarrow \infty} \frac{D_{n}\left(\frac{S}{4 n}, \frac{T}{2 n+1+\alpha}, \alpha\right)}{D_{n}(0,0, \alpha)},
$$

we find, as $n \rightarrow \infty$,

$$
\begin{aligned}
\frac{P_{n}\left(0 ; \frac{S}{4 n}, \frac{T}{2 n+1+\alpha}, \alpha\right)}{P_{n}(0 ; 0,0, \alpha)} & \sim \frac{\Delta(S, T, \alpha+1)}{\Delta(S, T, \alpha)} \\
& \sim \exp \left[C_{j}\left(\frac{T}{S}, \alpha+1\right)-C_{j}\left(\frac{T}{S}, \alpha\right)\right]+\cdots
\end{aligned}
$$

Note that (3.18) results from (3.12), (3.14) and (3.16) for $j=1,2,3$, and obviously the subsequent terms of it can be written out explicitly. 
We shall apply logarithmic potential theory with an external field in the next section to deduce an approximation of $P_{n}\left(0 ; \frac{S}{4 n}, \frac{T}{2 n+1+\alpha}, \alpha\right)$ for large $n$ and hence get the asymptotic formula for $P_{n}\left(0 ; \frac{S}{4 n}, \frac{T}{2 n+1+\alpha}, \alpha\right) / P_{n}(0 ; 0,0, \alpha)$. By comparing the result with (3.18), we find

$$
C_{j}\left(\frac{T}{S}, \alpha+1\right)-C_{j}\left(\frac{T}{S}, \alpha\right)=\log \left(\frac{\Gamma(1+\alpha)}{\sqrt{2 \pi}}\right)+\frac{T}{2 S}, \quad j=1,2,3 .
$$

\section{The Evaluation of $P_{n}(z ; s, t, \alpha)$ at $z=0$ via Logarithmic Potential Theory with an External Field}

Denote by $\left\{x_{k}\right\}_{k=1}^{n}$ the eigenvalues of the unitary ensembles of $n \times n$ Hermitian matrices with the weight function

$$
w(x ; t, \alpha)=x^{\alpha} \mathrm{e}^{-x-\frac{t}{x}}, \quad x \in[0, \infty), \alpha>-1, t \geq 0,
$$

and with the potential

$$
v(x)=-\log w(x ; t, \alpha)=-\alpha \log x+x+\frac{t}{x},
$$

and we suppose $x_{k} \geq s$ for $k=1,2, \ldots, n$. If we interpret $\left\{x_{k}\right\}_{k=1}^{n}$ as the positions of $n$ identically charged particles, then, for sufficiently large $n$, the particles can be approximated as a continuous fluid with a density $\sigma(x)$. Since $v(x)$ is convex for $x \in[s, \infty)$, we know that $\sigma(x)$ is supported on a single interval $[s, b]$. See [10] for a detailed analysis.

In Dyson's works [19], such $\sigma(x)$ is determined via the constrained minimization

$$
\min _{\sigma} F[\sigma] \text { subject to } \int_{s}^{b} \sigma(x) d x=n,
$$

with

$$
F[\sigma]:=\int_{s}^{b} \sigma(x) v(x) d x-\int_{s}^{b} \int_{s}^{b} \sigma(x) \log |x-y| \sigma(y) d x d y .
$$

According to Theorem 1.3 in Chapter I.1 of [31], the equilibrium density $\sigma(x)$ satisfies the condition

$$
v(x)-2 \int_{s}^{b} \log |x-y| \sigma(y) d y=A, \quad x \in[s, b],
$$

where $A$ is the Lagrange multiplier that fixes $\int_{s}^{b} \sigma(x) d x=n$. Differentiation of this equation with respect to $x$ gives rise to a singular integral equation

$$
2 P \int_{s}^{b} \frac{\sigma(y) d y}{x-y}=v^{\prime}(x), \quad x \in[s, b]
$$

where $P$ denotes the Cauchy principal value. Based on the theory of singular integral equations (see Theorem 3.1 in Chapter IV.3, [31]), we find

$$
\sigma(x)=\frac{1}{2 \pi^{2}} \sqrt{\frac{b-x}{x-s}} P \int_{s}^{b} \frac{v^{\prime}(y)}{y-x} \sqrt{\frac{y-s}{b-y}} d y, \quad x \in(s, b) .
$$


From which the normalization condition $\int_{s}^{b} \sigma(x) d x=n$ reads

$$
\frac{1}{2 \pi} \int_{s}^{b} \sqrt{\frac{y-s}{b-y}} v^{\prime}(y) d y=n .
$$

It should be pointed out that (4.1) and (4.2) are not applicable in the case $s=0$. Hence in our subsequent considerations, we assume $s>0$ and and therefore $S>0$.

Noting that

$$
v^{\prime}(x)=-\frac{\alpha}{x}+1-\frac{t}{x^{2}},
$$

with the aid of integral identities listed in the Appendix, we obtain from (4.1)

$$
\sigma(x)=\frac{1}{2 \pi} \sqrt{\frac{b-x}{x-s}}\left[1+\left(\frac{t}{2 \sqrt{s b}}-\left(\alpha+\frac{t}{2 b}\right) \sqrt{\frac{s}{b}}\right) \frac{1}{x}-\sqrt{\frac{s}{b}} \cdot \frac{t}{x^{2}}\right] .
$$

Here we require $\sqrt{s b}-\frac{t}{2}\left(\frac{1}{s}+\frac{1}{b}\right)>\alpha$ to guarantee that $\sigma(x)>0$ for $s<x<b$. Substituting (4.3) into the normalization condition $\int_{s}^{b} \sigma(x) d x=n$, or by using (4.2) directly, we find for $s>0$,

$$
\frac{b-s}{4}-\frac{\alpha}{2}+\frac{1}{2 \sqrt{s b}}\left(\alpha s+\frac{t}{2}\left(\frac{s}{b}-1\right)\right)=n .
$$

Clearing the square root, we come to the next lemma.

Lemma 4.1. For $s>0$ and $t \geq 0$, the upper edge $b$ of the support of the equilibrium density satisfies an algebraic equation of degree five

$$
b^{3}(b-2(2 n+\alpha)-s)^{2}=s\left(\left(2 \alpha-\frac{t}{s}\right) b+t\right)^{2} .
$$

Substituting $b=2(2 n+\alpha)+s+\tilde{b}$ into (4.4), one sees that, by sending $n \rightarrow \infty, \tilde{b}=O\left(n^{-1 / 2}\right)$. Hence, for large $n$, we suppose $b$ has the expansion of the form

$$
b=2(2 n+\alpha)+s+\sum_{j=1}^{\infty} d_{j}(s, t) n^{-\frac{j}{2}} .
$$

Plugging this into (4.4) and by comparing the coefficient of the highest order term in $n$, we get $d_{1}(s, t)= \pm\left(\alpha \sqrt{s}-\frac{t}{2 \sqrt{s}}\right)$. According to (3.19) of [27], we know that $d_{1}(s, 0)=-a \sqrt{s}$. Hence we choose $d_{1}(s, t)=\frac{t}{2 \sqrt{s}}-\alpha \sqrt{s}$. Successive coefficients $d_{j}(s, t)$ are uniquely determined, which ultimately gives the expansion of $b$ for large $n$.

Lemma 4.2. For $s>0$ and $t \geq 0$, the following asymptotic expansion of $b$ holds

$$
\begin{aligned}
b= & 4 n+2 \alpha+s+\left(\frac{t}{2 \sqrt{s}}-\alpha \sqrt{s}\right) n^{-\frac{1}{2}} \\
& +\frac{1}{8}\left(\alpha s^{\frac{3}{2}}+\left(2 \alpha^{2}-\frac{3 t}{2}\right) \sqrt{s}-\frac{\alpha t}{\sqrt{s}}\right) n^{-\frac{3}{2}} \\
& -\frac{1}{8}\left(\alpha^{2} s-\alpha t+\frac{t^{2}}{4 s}\right) n^{-2}+O\left(n^{-\frac{5}{2}}\right), \quad n \rightarrow \infty,
\end{aligned}
$$


Shulin Lyu et al. / The Hankel determinant associated with a singularly perturbed Laguerre unitary ensemble

from which, on replacing s by $\frac{S}{4 n}$ and t by $\frac{T}{2 n+1+\alpha}$, and by sending $n$ to $\infty$, we find

$$
\begin{aligned}
b= & 4 n+2 \alpha+\left(\frac{S}{4}-\frac{\alpha}{2} \sqrt{S}+\frac{T}{2 \sqrt{S}}\right) n^{-1} \\
& +\left(\frac{\alpha^{2}}{8} \sqrt{S}-\frac{(3 \alpha+2) T}{8 \sqrt{S}}\right) n^{-2}+O\left(n^{-3}\right), \quad n \rightarrow \infty .
\end{aligned}
$$

Based on logarithmic potential theory with an external field and the linear statistics formula, it is proved in [14] that the monic polynomial $P_{n}(x ; s, t, \alpha)$ orthogonal with respect to $w(x ; t, \alpha)$ over $[s, \infty)$ can be approximated as follows:

$$
P_{n}(z ; s, t, \alpha) \sim \mathrm{e}^{-S_{1}(z ; s, t, \alpha)-S_{2}(z ; s, t, \alpha)}, \quad z \in \mathbb{C} \backslash[s, b],
$$

where

$$
\begin{aligned}
S_{1}(z ; s, t, \alpha)= & \frac{1}{4} \log \left[\frac{16(z-s)(z-b)}{(b-s)^{2}}\left(\frac{\sqrt{z-s}-\sqrt{z-b}}{\sqrt{z-s}+\sqrt{z-b}}\right)^{2}\right], \\
S_{2}(z ; s, t, \alpha)= & -n \log \left(\frac{\sqrt{z-s}+\sqrt{z-b}}{2}\right)^{2} \\
& +\frac{1}{2 \pi} \int_{s}^{b} \frac{v(x) d x}{\sqrt{(b-x)(x-s)}}\left[\frac{\sqrt{(z-s)(z-b)}}{x-z}+1\right],
\end{aligned}
$$

and $v(x)=-\log w(x ; t, \alpha)=-\alpha \log x+x+\frac{t}{x}$. We point out here a simpler representation for $\mathrm{e}^{-S_{1}(z ; s, t, \alpha)}$ :

$$
\mathrm{e}^{-S_{1}(z ; s, t, \alpha)}=\frac{1}{2}\left[\left(\frac{z-b}{z-s}\right)^{\frac{1}{4}}+\left(\frac{z-s}{z-b}\right)^{\frac{1}{4}}\right] .
$$

The above result also appeared in the context of one dimensional free probability [26]. We now give an evaluation of $P_{n}(0 ; s, t, \alpha)$.

Theorem 4.1. The monic polynomials $P_{n}(x ; s, t, \alpha)$ orthogonal with respect to $x^{\alpha} \mathrm{e}^{-x-\frac{t}{x}}$ over $[s, \infty)$ with $\alpha>-1, t \geq 0, s>0$, is evaluated at $x=0$ by

$$
\begin{aligned}
(-1)^{n} P_{n}(0 ; s, t, \alpha) \sim & n^{n+\frac{\alpha}{2}+\frac{1}{4}}(4 s)^{-\frac{\alpha}{2}-\frac{1}{4}}\left(1+(1+2 \alpha) \frac{\sqrt{s}}{2 \sqrt{n}}+O\left(n^{-1}\right)\right) \\
& \cdot \exp \left[-n+\sqrt{4 n s}-\frac{s}{2}+\frac{t}{4 s}+O\left(n^{-\frac{1}{2}}\right)\right], \quad n \rightarrow \infty,
\end{aligned}
$$

so that, we have for $s=\frac{S}{4 n}$ and $t=\frac{T}{2 n+1+\alpha}$,

$$
\begin{aligned}
(-1)^{n} P_{n}\left(0 ; \frac{S}{4 n}, \frac{T}{2 n+1+\alpha}, \alpha\right) \sim & n^{n+\alpha+\frac{1}{2}} S^{-\frac{\alpha}{2}-\frac{1}{4}}\left(1+(1+2 \alpha)\left(\frac{\sqrt{S}}{4}+\frac{\alpha}{8}\right) n^{-1}+O\left(n^{-2}\right)\right) \\
& \cdot \exp \left[-n+\sqrt{S}+\frac{T}{2 S}+O\left(n^{-1}\right)\right], \quad n \rightarrow \infty .
\end{aligned}
$$

As a consequence, we obtain as $n \rightarrow \infty$,

$$
\frac{P_{n}\left(0 ; \frac{S}{4 n}, \frac{T}{2 n+1+\alpha}, \alpha\right)}{P_{n}(0 ; 0,0, \alpha)} \sim \frac{\Gamma(1+\alpha)}{\sqrt{2 \pi}} S^{-\frac{\alpha}{2}-\frac{1}{4}} \mathrm{e}^{\sqrt{S}+\frac{T}{2 S}} .
$$


Proof. In what follows, the symbol $\sim$ refers to 'asymptotic to' for large $n$, in the sense that the ratio tends to 1 . We have

$$
P_{n}(0 ; s, t, \alpha) \sim \mathrm{e}^{-S_{1}(0 ; s, t, \alpha)-S_{2}(0 ; s, t, \alpha)}
$$

where

$$
\mathrm{e}^{-S_{1}(0 ; s, t, \alpha)}=\frac{1}{2}\left[\left(\frac{b}{s}\right)^{\frac{1}{4}}+\left(\frac{s}{b}\right)^{\frac{1}{4}}\right]
$$

and

$$
\begin{aligned}
S_{2}(0 ; s, t, \alpha)= & -n \log \left(\frac{\sqrt{-s}+\sqrt{-b}}{2}\right)^{2} \\
& +\frac{1}{2 \pi} \int_{s}^{b} \frac{-\alpha \log x+x+\frac{t}{x}}{\sqrt{(b-x)(x-s)}}\left(\frac{\sqrt{(-s)(-b)}}{x}+1\right) d x .
\end{aligned}
$$

By using the integral formulas given in the Appendix and taking the branch $-s=s \mathrm{e}^{\pi i}$ and $-b=$ $b \mathrm{e}^{\pi i}$, we arrive at

$$
\mathrm{e}^{-S_{2}(0 ; s, t, \alpha)}=(-1)^{n} 4^{-n-\alpha}(\sqrt{b}+\sqrt{s})^{2 n}\left(\left(\frac{b}{s}\right)^{\frac{1}{4}}+\left(\frac{s}{b}\right)^{\frac{1}{4}}\right)^{2 \alpha} \cdot \exp \left[\left(\frac{t}{s b}-1\right)\left(\frac{\sqrt{b}-\sqrt{s}}{2}\right)^{2}\right]
$$

By plugging the expansion of $b$ given by (4.6) into $\mathrm{e}^{-S_{1}(0 ; s, t, \alpha)}$ and $\mathrm{e}^{-S_{2}(0 ; s, t, \alpha)}$, we obtain (4.7). Upon substituting $\frac{S}{4 n}$ for $s$ and $\frac{T}{2 n+1+\alpha}$ for $t$ in (4.7), and by sending $n$ to $\infty$, expression (4.8) follows.

Since $D_{n}(0,0, \alpha)$ has a closed-form expression and reads (see [29], p.321)

$$
D_{n}(0,0, \alpha)=\prod_{j=0}^{n-1} \Gamma(j+1) \Gamma(j+\alpha+1)
$$

we have

$$
(-1)^{n} P_{n}(0 ; 0,0, \alpha)=\frac{D_{n}(0,0, \alpha+1)}{D_{n}(0,0, \alpha)}=\frac{\Gamma(n+1+\alpha)}{\Gamma(1+\alpha)} .
$$

Hence, it follows from (4.8) that

$$
\begin{aligned}
\frac{P_{n}\left(0 ; \frac{S}{4 n}, \frac{T}{2 n+1+\alpha}, \alpha\right)}{P_{n}(0 ; 0,0, \alpha)} & \sim \Gamma(1+\alpha) \frac{n^{n+\alpha+\frac{1}{2}} \mathrm{e}^{-n}}{\Gamma(n+1+\alpha)} S^{-\frac{\alpha}{2}-\frac{1}{4}} \mathrm{e}^{\sqrt{S}+\frac{T}{2 S}} \\
& \sim \frac{\Gamma(1+\alpha)}{\sqrt{2 \pi}} S^{-\frac{\alpha}{2}-\frac{1}{4}} \mathrm{e}^{\sqrt{S}+\frac{T}{2 S}} .
\end{aligned}
$$

Here in the last step, we make use of the approximation

$$
\left(\frac{n}{\mathrm{e}}\right)^{n} \sim \frac{\Gamma(n+1+\alpha)}{\sqrt{2 \pi}} n^{-\alpha-\frac{1}{2}},
$$

which results from Stirling's formula [25]

$$
n ! \sim \sqrt{2 \pi n}\left(\frac{n}{\mathrm{e}}\right)^{n}
$$


Shulin Lyu et al. / The Hankel determinant associated with a singularly perturbed Laguerre unitary ensemble

and the standard asymptotic approximation for Gamma function

$$
\Gamma(n+\alpha) \sim \Gamma(n) n^{\alpha}, \quad \alpha \in \mathbb{C} .
$$

The proof is completed.

Bearing in mind that

$$
\frac{P_{n}\left(0 ; \frac{S}{4 n}, \frac{T}{2 n+1+\alpha}, \alpha\right)}{P_{n}(0 ; 0,0, \alpha)} \sim \frac{\Delta(S, T, \alpha+1)}{\Delta(S, T, \alpha)}, \quad n \rightarrow \infty,
$$

we establish the following statement.

Corollary 4.1. The undetermined terms that appear in the asymptotic expansions of $\log \Delta(S, T, \alpha)$ in the previous section satisfy a difference equation

$$
C_{j}\left(\frac{T}{S}, \alpha+1\right)-C_{j}\left(\frac{T}{S}, \alpha\right)=\log \left(\frac{\Gamma(1+\alpha)}{\sqrt{2 \pi}}\right)+\frac{T}{2 S}, \quad j=1,2,3,
$$

and therefore, up to a constant that is independent of $\alpha, S$ and $T$ the term $C_{j}\left(\frac{T}{S}, \alpha\right)$ has the form

$$
\log \frac{G(\alpha+1)}{(2 \pi)^{\alpha / 2}}+\frac{\alpha}{2} \cdot \frac{T}{S}
$$

where $G(\cdot)$ denotes the Barnes-G function defined by

$$
G(z+1)=\Gamma(z) G(z), \quad G(1)=1 .
$$

Proof. Since the proofs of (4.10) for $j=1,2,3$ are similar, we only prove the case $j=1$ with $S$ large and $T \geq 0$ fixed. We find from (3.12)

$$
\begin{aligned}
\lim _{n \rightarrow \infty} & \frac{P_{n}\left(0 ; \frac{S}{4 n}, \frac{T}{2 n+1+\alpha}, \alpha\right)}{P_{n}(0 ; 0,0, \alpha)}=\frac{\Delta(S, T, \alpha+1)}{\Delta(S, T, \alpha)} \\
& \sim \exp \left[C_{1}\left(\frac{T}{S}, \alpha+1\right)-C_{1}\left(\frac{T}{S}, \alpha\right)+\sqrt{S}-\left(\frac{\alpha}{2}+\frac{1}{4}\right) \log S\right] .
\end{aligned}
$$

Comparing this with (4.9) leads to

$$
C_{1}\left(\frac{T}{S}, \alpha+1\right)-C_{1}\left(\frac{T}{S}, \alpha\right)=\log \left(\frac{\Gamma(1+\alpha)}{\sqrt{2 \pi}}\right)+\frac{T}{2 S} .
$$

\section{Acknowledgement}

The financial support of the Macau Science and Technology Development Fund under grant number FDCT 130/2014/A3 and FDCT 023/2017/A1 is gratefully acknowledged. We would also like to thank the University of Macau for generous support: MYRG 2014-00011 FST, MYRG 2014-00004 FST. 
Shulin Lyu et al. / The Hankel determinant associated with a singularly perturbed Laguerre unitary ensemble

\section{Appendix A. Some Relevant Integral Identities}

We state the following integral identities for $0<a<b$, which are relevant to our derivation and could be found in [9], [15] and [20].

$$
\begin{aligned}
\int_{a}^{b} \frac{d x}{\sqrt{(b-x)(x-a)}} & =\pi \\
\int_{a}^{b} \frac{x d x}{\sqrt{(b-x)(x-a)}} & =\frac{(a+b) \pi}{2} \\
\int_{a}^{b} \frac{d x}{x \sqrt{(b-x)(x-a)}} & =\frac{\pi}{\sqrt{a b}} \\
\int_{a}^{b} \frac{d x}{x^{2} \sqrt{(b-x)(x-a)}} & =\frac{(a+b) \pi}{2(a b)^{3 / 2}} \\
\int_{a}^{b} \frac{\log x d x}{\sqrt{(b-x)(x-a)}} & =2 \pi \log \left(\frac{\sqrt{a}+\sqrt{b}}{2}\right) \\
\int_{a}^{b} \frac{\log x d x}{x \sqrt{(b-x)(x-a)}} & =\frac{2 \pi}{\sqrt{a b}} \log \left(\frac{2 \sqrt{a b}}{\sqrt{a}+\sqrt{b}}\right) .
\end{aligned}
$$

\section{References}

[1] E. Basor and Y. Chen, Painlevé V and the distribution function of a discontinuous linear statistic in the Laguerre unitary ensembles, J. Phys. A: Math. Theor. 42 (2009), 035203 (18pp).

[2] E. Basor and Y. Chen, Perturbed Laguerre unitary ensembles, Hankel determinants, and information theory, Math. Meth. Appl. Sci. 38 (2015), 4840-4851.

[3] E. Basor, Y. Chen and T. Ehrhardt, Painlevé V and time-dependent Jacobi polynomials, J. Phys. A: Math. Theor. 43 (2010), 015204 (25pp).

[4] E. Basor, Y. Chen and L. Zhang, PDEs satisfied by extreme eigenvalues distributions of GUE and LUE, Random Matrices: Theory and Applications, 1 (2012), 1150003 (21pp).

[5] M. Chen and Y. Chen, Singular linear statistics of the Laguerre unitary ensemble and Painlevé III. Double scaling analysis, J. Math. Phys. 56 (2015), 063506 (14pp).

[6] M. Chen, Y. Chen and E. Fan, Perturbed Hankel determinant, correlation functions and Painlevé equations, J. Math. Phys. 57 (2016), 023501 (31pp).

[7] Y. Chen and D. Dai, Painlevé V and a Pollaczek-Jacobi type orthogonal polynomials, J. Approx. Theory 162 (2010), 2149-2167.

[8] Y. Chen and M.V. Feigin, Painlevé IV and degenerate Gaussian unitary ensembles, J. Phys. A: Math. Gen. 39 (2006), 12381-12393.

[9] Y. Chen, N.S. Haq and M.R. McKay, Random matrix models, double-time Painlevé equations, and wireless relaying, J. Math. Phys. 54 (2013), 063506 (55pp).

[10] Y. Chen, and M.E.H. Ismail, Thermodynamic relations of the Hermitian matrix ensembles, J. Phys. A: Math. Gen. 30 (1997), 6633-6654.

[11] Y. Chen and M.E.H. Ismail, Ladder operators and differential equations for orthogonal polynomials, $J$. Phys. A: Math. Gen. 30 (1997), 7817-7829.

[12] Y. Chen and M. Ismail, Jacobi polynomials from compatibility conditions, Proc. Amer. Math. Soc. 133 (2005), 465-472.

[13] Y. Chen and A. Its, Painlevé III and a singular linear statistics in Hermitian random matrix ensembles, I, J. Approx. Theory 162 (2010), 270-297.

[14] Y. Chen and N. Lawrence, On the linear statistics of Hermitian random matrices, J. Phys. A: Math. Gen. 31 (1998), 1141-1152. 
Shulin Lyu et al. / The Hankel determinant associated with a singularly perturbed Laguerre unitary ensemble

[15] Y. Chen and M.R. McKay, Coulumb fluid, Painlevé transcendents, and the information theory of MIMO systems, IEEE Trans. Inf. Theory 58 (2012), 4594-4634.

[16] Y. Chen and G. Pruessner, Orthogonal polynomials with discontinuous weights, J. Phys. A: Math. Gen. 38 (2005), L191-L198.

[17] Y. Chen and L. Zhang, Painlevé VI and the unitary Jacobi ensembles, Stud. Appl. Math. 125 (2010), 91-112.

[18] D. Dai and L. Zhang, Painlevé VI and Hankel determinants for the generized Jacobi weight, J. Phys. A: Math. Theor. 43 (2010), 055207 (14pp).

[19] F.J. Dyson, Statistical theory of the energy levels of complex systems. I, II and III, J. Math. Phys. 3 (1962), 140-156, 157-165, 166-175.

[20] I.S. Gradshteyn and I.M. Ryzhik, Table of Integrals, Series, and Products 8th ed., (Academic press, 2014).

[21] N.J. Higham, Functions of Matrices: Theory and Computation, (SIAM, 2008).

[22] L.K. Hua, Harmonic Analysis of Functions of Several Complex Variables in the Classical Domains, (AMS, 1963).

[23] M.E.H. Ismail, Classical and Quantum Orthogonal Polynomials in One Variable, Encyclopedia of Mathematics and its Applications 98, (Cambridge University Press, 2005).

[24] M. Jimbo and T. Miwa, Monodromy perserving deformation of linear ordinary differential equations with rational coefficients. II, Physica $2 D$ (1981), 407-448.

[25] N.N. Lebedev, Special Functions \& Their Applications, (Dover Publications, INC., New York, 1972).

[26] M. Ledoux and I. Popescu, Mass transportation proofs of free functional inequalities, and free Poincaré inequalities, J. Funct. Anal. 257 (2009), 1175-1221.

[27] S. Lyu, Y. Chen and E. Fan, Asymptotic gap probability distributions of the Gaussian unitary ensembles and Jacobi unitary ensembles, Nucl. Phys. B 926 (2018), 639-670.

[28] A.P. Magnus, Painlevé-type differential equations for the recurrence coefficients of semi-classical orthogonal polynomials, J. Comput. Appl. Math. 57 (1995), 215-237.

[29] M. L. Mehta, Random Matrices 3rd ed., (Elsevier (Singapore) Pte Ltd., Singapore, 2006).

[30] C. Min and Y. Chen, Gap probability distribution of the Jacobi unitary ensemble: an elementary treatment, from finite $n$ to double scaling, Stud. Appl. Math. 140 (2018), 202-220.

[31] E.B. Saff and V. Totik, Logarithmic Potentials with External Fields, Grundlehren der mathematischen Wissenschaften 316, (Springer-Verlag Berlin Heidelberg GmbH, 1997).

[32] G. Szegö, Orthogonal Polynomials, (AMS, New York, 1939).

[33] C.A. Tracy and H. Widom, Level spacing distributions and the Bessel kernel, Commun. Math. Phys. 161 (1994), 289-309.

[34] S. Xu, D. Dai and Y. Zhao, Critical edge behavior and the Bessel to Airy transition in the singularly perturbed Laguerre unitary ensemble, Commun. Math. Phys. 332 (2014), 1257-1296. 